OPEN ACCESS

Edited by:

Jianhua Fan,

Carnegie Institution for Science (CIS),

United States

Reviewed by:

Wenqiang Yang,

Carnegie Institution for Science,

United States

Conrad Mullineaux

Queen Mary University of London,

United Kingdom

*Correspondence:

Olaf Kruse

olaf.kruse@uni-bielefeld.de

Specialty section: This article was submitted to

Plant Biotechnology,

a section of the journal

Frontiers in Plant Science

Received: 13 June 2017

Accepted: 19 July 2017

Published: 03 August 2017

Citation:

Venkanna $D$, Südfeld $C$, Baier $T$, Homburg SV, Patel AV, Wobbe L and Kruse O (2017) Knock-Down of the

IFR1 Protein Perturbs

the Homeostasis of Reactive

Electrophile Species and Boosts Photosynthetic Hydrogen Production

in Chlamydomonas reinhardtii.

Front. Plant Sci. 8:1347.

doi: 10.3389/fpls.2017.01347

\section{Knock-Down of the IFR1 Protein Perturbs the Homeostasis of Reactive Electrophile Species and Boosts Photosynthetic Hydrogen Production in Chlamydomonas reinhardtii}

\author{
Deepak Venkanna ${ }^{1}$, Christian Südfeld ${ }^{1}$, Thomas Baier ${ }^{1}$, Sarah V. Homburg ${ }^{2}$, \\ Anant V. Patel ${ }^{2}$, Lutz Wobbe ${ }^{1}$ and Olaf Kruse ${ }^{1 *}$
}

${ }^{1}$ Faculty of Biology, Center for Biotechnology (CeBiTec), Bielefeld University, Bielefeld, Germany, ${ }^{2}$ Faculty of Engineering and Mathematics, Fermentation and Formulation of Biologicals and Chemicals, Bielefeld University of Applied Sciences,

Bielefeld, Germany

The protein superfamily of short-chain dehydrogenases/reductases (SDR), including members of the atypical type (aSDR), covers a huge range of catalyzed reactions and in vivo substrates. This superfamily also comprises isoflavone reductase-like (IRL) proteins, which are aSDRs highly homologous to isoflavone reductases from leguminous plants. The molecular function of IRLs in non-leguminous plants and green microalgae has not been identified as yet, but several lines of evidence point at their implication in reactive oxygen species homeostasis. The Chlamydomonas reinhardtii $\mathrm{IRL}$ protein IFR1 was identified in a previous study, analyzing the transcriptomic changes occurring during the acclimation to sulfur deprivation and anaerobiosis, a condition that triggers photobiological hydrogen production in this microalgae. Accumulation of the cytosolic IFR1 protein is induced by sulfur limitation as well as by the exposure of $C$. reinhardtii cells to reactive electrophile species (RES) such as reactive carbonyls. The latter has not been described for IRL proteins before. Over-accumulation of IFR1 in the singlet oxygen response 1 (sor1) mutant together with the presence of an electrophile response element, known to be required for SOR1-dependent gene activation as a response to RES, in the promoter of IFR1, indicate that IFR1 expression is controlled by the SOR1dependent pathway. An implication of IFR1 into RES homeostasis, is further implied by a knock-down of IFR1, which results in a diminished tolerance toward RES. Intriguingly, IFR1 knock-down has a positive effect on photosystem II (PSII) stability under sulfurdeprived conditions used to trigger photobiological hydrogen production, by reducing PSIl-dependent oxygen evolution, in C. reinhardtii. Reduced PSIl photoinhibition in IFR1 knock-down strains prolongs the hydrogen production phase resulting in an almost doubled final hydrogen yield compared to the parental strain. Finally, IFR1 
knock-down could be successfully used to further increase hydrogen yields of the high hydrogen-producing mutant stm6, demonstrating that IFR1 is a promising target for genetic engineering approaches aiming at an increased hydrogen production capacity of $C$. reinhardtii cells.

Keywords: Chlamydomonas reinhardtii, photobiological hydrogen production, isoflavone reductase-like proteins, short-chain dehydrogenases/reductases, reactive electrophile species, singlet oxygen response 1 (sor1)

\section{INTRODUCTION}

Among the most urgent challenges of our society today, are those associated to global warming, depletion of fossil fuels and a steady increase of the energy demand, which can pose a threat to economic and political stability (Organisation for Economic Co-operation and Development [OECD]/International Energy Agency [IEA], 2011). Photosynthesis-driven $\mathrm{H}_{2}$ production by photosynthetic microbes, such as cyanobacteria and microalgae, has a perfect carbon footprint, because of its zero $\mathrm{CO}_{2}$ emission. Within photobiological hydrogen production electrons and protons from water splitting are directed via photosynthesis toward specific $\mathrm{H}_{2}$-evolving enzymes, the hydrogenases (Gaffron and Rubin, 1942). Microalgae exploit Fe-Fe hydrogenases, which compared to other hydrogenases are highly efficient because of their extraordinarily high turnover number (Volgusheva et al., 2013; Lubitz et al., 2014). However, due to its oxygen sensitivity (Ghirardi et al., 1997), oxygenic photosynthesis cannot be directly coupled to hydrogen production in green microalgae. Therefore, photobiological hydrogen production has to be split into a two-stage process, which can be achieved by the experimental protocol proposed by Melis et al. (2000). This protocol relies on biomass generation under sulfur-replete conditions in the first stage and subsequent withdrawal of sulfur to trigger photoinhibition of photosystem II, resulting in a continuous decline of photosynthetic oxygen evolution, while mitochondrial respiration remains relatively unaffected by the lack of sulfur in the medium. In sealed culture flasks, this cultivation regime helps establishing anaerobic conditions, which are a prerequisite for the induction of the hydrogenase pathway (Ghysels and Franck, 2010). In Chlamydomonas reinhardtii, sulfur deprivation results in a strong down-regulation of the Calvin cycle and photosynthetic light reactions, based on a rapid decrease of Rubisco levels (Zhang et al., 2002) and an impaired PSII repair cycle, which relies on the de novo synthesis of the PSII subunit D1, which is restricted by the limited availability of sulfur-containing amino acids under these conditions (Wykoff et al., 1998). Although a massive decline in water-splitting activity is a prerequisite for the establishment of anaerobic conditions, which enable hydrogen production via the oxygen-sensitive hydrogenase enzyme, several studies clearly demonstrated that residual PSII activity and linear electron transport toward the hydrogenase are indispensable for efficient hydrogen production in C. reinhardtii (Antal et al., 2003; Volgusheva et al., 2013; Baltz et al., 2014; Steinbeck et al., 2015). The C. reinhardtii mutant stm6 (Schönfeld et al., 2004) displays an enhanced hydrogen production capacity
(Kruse et al., 2005) and its increased rate of mitochondrial oxygen consumption (Uhmeyer et al., 2017), was proposed to protect PSII during sulfur deprivation by accelerating the establishment of anaerobic conditions (Volgusheva et al., 2013), where irreversible, oxygen-dependent photoinhibition (Vass et al., 1992) cannot occur. Besides the PSII-dependent pathway of hydrogen production, starch degradation and subsequent glycolysis can provide $\mathrm{NADH}$, which can be used to feed electrons into the photosynthetic electron transport chain without the need for water-splitting at PSII (Chochois et al., 2009; Baltz et al., 2014). Therefore, larger starch reserves present in stm6 compared to wild type strains also contribute to the higher hydrogen production capacity seen for this mutant (Kruse et al., 2005; Doebbe et al., 2010). Cyclic electron flow (CEF) around photosystem I competes with electron delivery to the hydrogenase and a reduced CEF activity of stm6 is another important aspect of its phenotype, which should significantly contribute to the elevated hydrogen production potential (Kruse et al., 2005). In addition to its photobiological production, hydrogen can also be produced under dark fermentative conditions in C. reinhardtii (Grossman et al., 2011).

With the aim to generate $C$. reinhardtii strains producing increased amounts of hydrogen upon exposure to sulfur limitation, several strategies have already been applied, which mainly targeted the oxygen sensitivity of the hydrogenase, the competition between CEF and hydrogen production, the efficiency of light conversion in the antenna and cellular starch contents (for review see Dubini and Ghirardi, 2015). Transcriptomics conducted with $C$. reinhardtii cells subjected to hydrogen production conditions could be another strategy to identify novel gene targets for the optimization of hydrogen production via genetic engineering (Nguyen et al., 2011; Toepel et al., 2013).

In a previous study (Nguyen et al., 2011), a transcript encoding the protein IFR1 (Cre11.g477200; NmrA-like) accumulated strongly in hydrogen-producing cells of C. reinhardtii. NmrA-like proteins belong to the protein superfamily of atypical shortchain dehydrogenases/reductases (aSDRs), which also contains isoflavone reductase-like (IRL) proteins (Moummou et al., 2012). IRL proteins from higher plants such as OsIRL from rice, were proposed to be implicated in ROS homeostasis, as OsIRL expression is induced by ROS and an overexpression confers enhanced ROS tolerance (Kim et al., 2010). SDRs and aSDRs including IRL proteins remain poorly characterized in microalgae thus far (Moummou et al., 2012). Therefore, we analyzed the function of IFR1 by applying a forward genetics strategy based 
on the use of artificial microRNA (amiRNA)-mediated knockdown of IFR1 in two distinct $C$. reinhardtii strains and subsequent analysis of the resulting phenotype, with a special focus on photosynthetic hydrogen production.

\section{MATERIALS AND METHODS}

\section{Chemicals}

3-(3,4-Dichlorophenyl)-1,1-dimethylurea (DCMU), 2,5-Dibromo-6-isopropyl-3-methyl-1,4-Benzoquinone (DBMIB), 2EHexenal, Hydrogen peroxide $\left(\mathrm{H}_{2} \mathrm{O}_{2}\right)$, Methyl Viologen (MV), Neutral red (NR), and Rose Bengal (RB) were purchased from Sigma-Aldrich.

\section{Strains and Growth Conditions}

Chlamydomonas reinhardtii wild type CC124 (137c mt-), 4A+ (137c background) and mutant CC4604- sor1 (mt+) (Fischer et al., 2012) were obtained from the Chlamydomonas Center. UVM4, a UV mutant derived from CC4350 (cw15 arg7-8 mt+) known to efficiently express nuclear transgenes (Neupert et al., 2009) was kindly provided by R. Bock (MPI for Molecular Plant Physiology, Potsdam-Golm). The MOC1 knock-out mutant stm6 was generated via random insertion of plasmid pArg7.8 (Debuchy et al., 1989), carrying the Arg7 gene, into the nuclear genome of the arginine auxotrophic strain, CC1618. The MOC1-complemented strain B13 (Schönfeld et al., 2004) was generated by co-transforming stm6 with a 37-kb Moc1containing cosmid isolated from a cosmid library and the Cry 1 gene as a dominant selectable marker conferring resistance to emetine (plasmid p613; Nelson et al., 1994). All strains were grown photoheterotrophically in TAP (tris acetate phosphate) medium (Harris, 1989) at $25^{\circ} \mathrm{C}$ with continuous white light of $100 \mu \mathrm{mol} \mathrm{m} \mathrm{m}^{-2} \mathrm{~s}^{-1}$. Experiments were performed by using the cells from mid-log phase. For hydrogen production, cells were harvested and washed three times with TAP-S medium. The cells were finally suspended in TAP-S to the tune of $\sim 25 \mu \mathrm{g} / \mathrm{ml}$ of chlorophyll. Hydrogen setup and gas measurement was carried out as described previously (Doebbe et al., 2010). After $20 \mathrm{~h}$ of anaerobic conditions, the effect of DCMU on $\mathrm{H}_{2}$ production was assessed by adding $20 \mu \mathrm{M}$ DCMU to the sealed bioreactors. Quantitative analysis of RES and ROS stress tolerance was evaluated by growing $2 \times 10^{6}$ cells $/ \mathrm{ml}$ in TAP at $100 \mu \mathrm{mol} \mathrm{m} \mathrm{m}^{-2} \mathrm{~s}^{-1}$ with following chemicals: $5 \mu \mathrm{M}$ DBMIB, $500 \mu \mathrm{M} 2 E$-Hexenal, $4 \mu \mathrm{M}$ RB, $15 \mu \mathrm{M}$ NR, $0.5 \mu \mathrm{M} \mathrm{MV}$ and $7 \mathrm{mM} \mathrm{H} \mathrm{O}_{2}$. Cell growth was determined by analyzing $\mathrm{OD}_{680}$ and cell count (Z2 cell and particle counter, Beckman Coulter) at 0 and $24 \mathrm{~h}$ and $10 \mu \mathrm{l}$ of culture was spotted on TAP agar plate for recovery.

\section{Generation of IRL Knock-Down Strains}

The artificial microRNA sequence for generating IRL knockdown was designed with a web based tool WMD3 ${ }^{1}$. The amiRNA sequences were generated to target exons 2 (forward: ctagtCAGGTCCAGGAGATTGATATAtctcgctgatcggcaccatg ggg-

${ }^{1}$ http://wmd3.weigelworld.org
gtggtggtgatcagcgcaTATAACAATCTCCTGGACCTGg; reverse: ctagcCAGGTCCAG GAGATTGTTATAtagcgctgatcaccaccacccc catggtgccgatcagcgagaTATATCAATCTCCTGGACCTGa) and 4 (forward: ctagtGAGCACGCTATTAAGGTCGTAtctcgctgatcgg caccatgg gggtggtggtgatcagcgctaTACGGTCTTA-ATAGCGTGCT $\mathrm{Cg}$ and reverse: ctagcGAGCACGC TATTAAGACCGTAtagcgct gatcaccaccaccccatggtgccgatcagcgagaTACGACCTTAATAG GT GCTCa) of the coding region and cloned into vector pChlamiRNAi3int (Molnar et al., 2009). CC124 was transformed by electroporation (Jaeger et al., 2017) and stm6 was transformed via glass beads as mentioned previously (Kindle, 1990). Transformants were selected on paromomycin $(10 \mu \mathrm{g} / \mathrm{ml})$ TAP agar plates and transferred to sulfur depleted medium for screening.

\section{Antibody and Recombinant Protein Production}

The polyclonal antiserum directed against a 17 aa polypeptide IFR1 was raised in rabbit (Agrisera, Sweden). To heterologously express IFR1 in Escherichia coli, a codon optimized full length IFR1 coding sequence (phytozome: Transcript Cre11.g477200.t1.2) was synthesized de novo (Genscript, United States) and cloned between the NdeI and XhoI restriction sites of expression vector $\mathrm{pET}-24 \mathrm{a}(+)$ (Novagen), enabling streptag-based affinity purification.

\section{RNA Extraction and Quantitative Real Time PCR}

Real-time RT-PCR was performed with DNaseI (RQ1 RNase -free DNase, Promega)-digested total RNA samples which was subjected to reverse transcription and PCR amplification using the SensiFAST ${ }^{\mathrm{TM}}$ SYBR No-ROX One-Step Kit (BIOLINE, Germany). SYBR Green I fluorescence was recorded on a DNA Engine Opticon (Bio-RAD, Germany). Per sample $100 \mathrm{ng}$ total RNA were used and RPL13 (Gene ID: 5718254) as well as RACK1 (GeneID: 5723548) served as housekeeping genes. The following primers were used within the study: IFR1 (5'-ATGGC GACTAAGAAGCACAC- $3^{\prime}$ and 5'-CGAAGCCTGCTCATTGT AGT-3'), RPL13 (5'-ATTCTTGCCGGGCAGCAGATTGTG-3' and $5^{\prime}$-TTGCGCAGGAAG CGGTCATACTTC-3') and RACK1 (5'-TCAACATCACCAGCAAGAAGG-3' and $5^{\prime}$-CTGGGCAT TTACAGGGAGTG-3'). Relative mRNA expression levels were calculated according to Pfaffl (Pfaffl, 2001).

\section{SDS-PAGE and Immunoblotting}

Cells were pelleted $(3000 \times \mathrm{g}, 3 \mathrm{~min})$ and suspended in lysis buffer (60 mM Tris pH 6.8, 2\% SDS, 10\% glycerol and freshly added $1 \mathrm{mM}$ Pefabloc). Total proteins were extracted via freezethaw cycle in liquid $\mathrm{N}_{2}$ and quantified by Lowry assay (BioRAD). The proteins were separated by a $12 \%$ Tris-glycine SDS-PAGE and blotted on to a nitrocellulose membrane. After overnight blocking (5\% Milk powder in TBST with $0.1 \%$ Tween), the membrane was incubated at room temperature for $1.5 \mathrm{~h}$ with IFR1-specific antiserum (1:2500), washed and then incubated for $1 \mathrm{~h}$ with a peroxidase-conjugated anti-rabbit antibody (Agrisera, Sweden) for chemiluminescence detection (ECL: GE Healthcare). 
Signals were visualized using the FUSION-FX7 detection system (Peqlab, Germany). Protein bands were quantified with MyImageAnalysis software (ThermoFisher Scientific).

\section{Chlorophyll Fluorescence Analyses}

To determine the maximum quantum yield $\left(\mathrm{F}_{v} / \mathrm{F}_{m}\right), 2 \mathrm{ml}$ samples of a culture were incubated in the dark and aerated for $20 \mathrm{~min}$. Chlorophyll fluorescence changes were recorded during a $10 \mathrm{~min}$ induction curve with actinic light $\left(800 \mu \mathrm{mol}\right.$ photons $\left.\mathrm{m}^{-2} \mathrm{~s}^{-1}\right)$ using a Mini PAM (Waltz) and $\mathrm{F}_{v} / \mathrm{F}_{m}$ calculated according to the following equation (Maxwell and Johnson, 2000):

$$
\frac{F_{\mathrm{v}}}{\mathrm{F}_{\mathrm{m}}}=\frac{\mathrm{F}_{\mathrm{m}}-\mathrm{F}_{0}}{\mathrm{~F}_{\mathrm{m}}}
$$

\section{Construction of Fusion Protein and Confocal Microscopy}

The IFR1 coding sequence, codon-optimized for the nuclear codon bias of $C$. reinhardtii was cloned into vector pOptmVenus_Paro (Lauersen et al., 2015) by using the NdeI::BglII and EcoRV::EcoRI restriction sites to obtain C-terminal and $\mathrm{N}$-terminal fusions, respectively. Fluorescence imaging was accomplished with a confocal laser scanning microscope (LSM780, Carl Zeiss GmbH, Germany) with specific filters for chlorophyll and mVenus as described before (Lauersen et al., 2015).

\section{Statistical Analysis}

The significance of results was evaluated with a student's twotailed $t$-test for independent samples. The significance threshold was set between $p<0.05$ to $p<0.1$. Error bars represent standard error (SE) and in case of box plots the whiskers represent variability within the first and third quartile.

\section{RESULTS}

\section{IFR1 Is an Atypical Short-Chain Dehydrogenase that Accumulates in the Cytosol of C. reinhardtii as a Response to Abiotic Stress}

A previous study (Nguyen et al., 2011), demonstrated that a transcript encoding a putative isoflavone reductase (IFR1) accumulated significantly in hydrogen-producing cells of C. reinhardtii. An NCBI-BLAST search using the amino acid sequence of IFR1 (Phytozome locus name Cre11.g477200; C. reinhardtii v5.5) revealed that this protein contains a conserved phenylcoumaran benzylic ether reductase (PCBER) like domain (specific hit/e-value $1.55 \mathrm{e}^{-68}$ ). PCBERs are NADPHdependent aromatic alcohol reductases, and are described as atypical members of the short-chain dehydrogenase/reductase (SDR) family (Min et al., 2003). Atypical SDRs possess an $\mathrm{N}$-terminus characteristic of $\mathrm{NAD}(\mathrm{P})$-binding proteins and a small C-terminal domain presumed to be involved in substrate binding (Filling et al., 2002; Persson et al., 2003; Kavanagh et al., 2008). In contrast to classical SDRs, they do not have the conserved active site tyrosine residue typically found in SDRs and contain an atypical glycine-rich NADP-binding motif reading GXGXXG or G[GA]XGXXG (Supplementary Figure S1). The amino acid sequence of IFR1 shows identities to other members of the protein family in the range of $20-30 \%$ (Supplementary Table S1), with the highest similarity found for isoflavone reductases (Babiychuk et al., 1995) and IRL proteins (Petrucco et al., 1996) (Supplementary Figure S1 and Table S1).

The C. reinhardtii IFR1 gene encodes a $32 \mathrm{kDa}$ protein whose localization was predicted to be cytosolic by the in silico prediction tool PredAlgo (Tardif et al., 2012). To confirm that IFR1 indeed resides in the cytosol, IFR1 was C- and N-terminally fused with YFP (mVenus variant; Kremers et al., 2006) and expressed in the $C$. reinhardtii cell line UVM4 (Neupert et al., 2009). Two strains, stably expressing either full length IFR1YFP (C) or IFR1-YFP (N) were identified via immunoblots (Supplementary Figure S2; C and N). YFP fluorescence could be detected in both strains expressing YFP, either N- or C- terminally fused to IFR1 (Figure 1; N and C), while the parental control strain (PCS) only emitted red chlorophyll auto-fluorescence from the cup-shaped structure representing the chloroplast. Superimposition of the chlorophyll and YFP fluorescence demonstrated that the YFP-tagged IFR1 variants displayed a distribution of the YFP signal identical to that observed in the control strain (Cyto), expressing YFP in the cytosol (Lauersen et al., 2015). YFP-tagging of IFR1 in conjunction with confocal laser-scanning microscopy demonstrated that the localization of IFR1 is indeed cytosolic.

In a previous study (Nguyen et al., 2011) we compared the transcriptomes of the $C$. reinhardtii wild type (wt) cc406 and the high hydrogen production mutant stm6glc4 (Doebbe et al., 2007) [derived from stm6 (Kruse et al., 2005)] during photosynthetic hydrogen production triggered by sulfur deprivation (Melis et al., 2000). The transcriptome data revealed that within the peak hydrogen production phase, IFR1 transcripts accumulated to a high extent ( $10-40$-fold compared to sulfur-replete condition) in both strains. In order to confirm that the accumulation of IFR1 transcript is indeed translated into elevated protein amounts, we analyzed protein samples taken at distinct points from a hydrogen-producing culture of a C. reinhardtii wild type (Figure 2A). A strong induction of IFR1 protein expression was observed in the wild type under S-deprived anaerobic $\mathrm{H}_{2}$ production conditions. IFR1 accumulation started before the onset of anaerobiosis and $\mathrm{H}_{2}$ production conditions (from $24 \mathrm{~h}$ onward), indicating that sulfur deprivation rather than anaerobiosis is required for IFR1 induction. An inspection of published RNAseq data sets using AlgaePath (Zheng et al., 2014) revealed that sulfur deprivation alone triggers IFR1 mRNA accumulation [ $\sim 8$-fold induction $6 \mathrm{~h}$ after withdrawal of sulfur; González-Ballester et al., 2010; gene expression omnibus (GEO) series GSE17970]. An even stronger induction was observed within a transcriptome study analyzing the modulation of the $C$. reinhardtii transcriptome in response to nitrogen depletion ( $\sim 46$-fold after 48 h; Miller et al., 2010; GSE24367), but IFR1 protein expression could not be detected under nitrogen-deplete conditions (data not shown), demonstrating that IFR1 accumulation is not generally observed as a response 


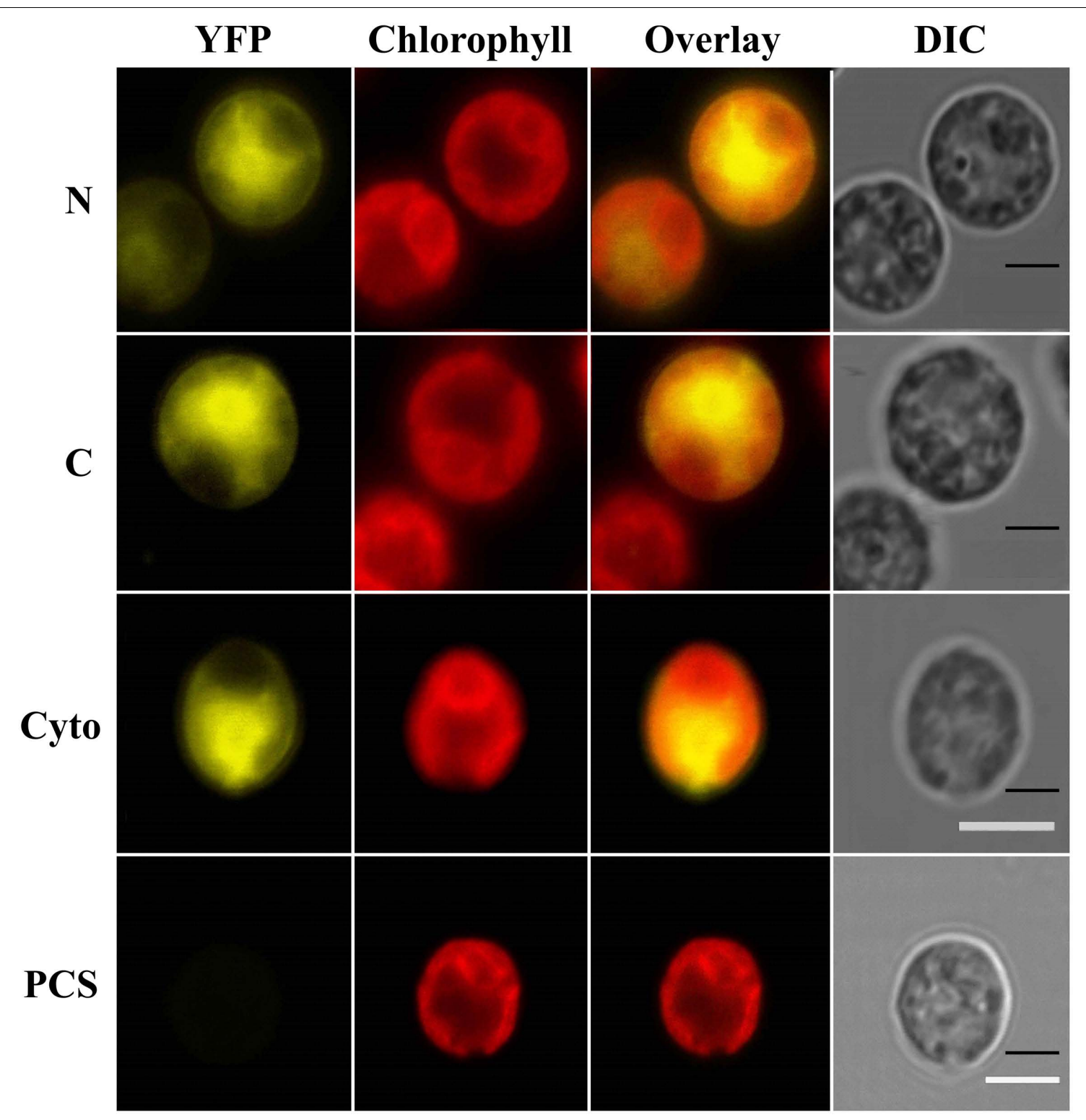

FIGURE 1 | IFR1 localizes to the cytosol in Chlamydomonas reinhardtii cells. Laser scanning confocal microscopy detection of subcellular localization of the mVenus (yellow) fluorescent reporter fused to N- or C-terminus of IFR1 (N/C). A cell line expressing mVenus in the cytosol (Cyto, Lauersen et al., 2015) and the parental strain (PCS) served as controls. Individual imaging channels are presented, YFP: mVenus reporter signal in the yellow range, Chlorophyll: autofluorescence of chlorophyll visualized in the red range and used to orient the cells, Overlay: YFP and Chloro channel overlay, DIC: differential interference contrast. Scale bars represent $5 \mu \mathrm{m}$.

to macronutrient limitation. While effects of carbon dioxide limitation on IFR1 transcript accumulation were comparably small ( 2-fold; Fang et al., 2012; GSE33927), exposure of wild type $C$. reinhardtii cells to hydrogen peroxide led to a rapid accumulation of IFR1 transcript $(\sim 19$-fold within 1 h; Blaby et al., 2015; GSE34826). In addition, a previous transcriptome study indicated that IFR1 belongs to the set of genes overexpressed in the mutant singlet oxygen resistant 1 (sor 1 ), which shows a constitutively higher expression of genes implicated in the detoxification of reactive oxygen and electrophile species [9.8 in sor 1 vs. 0 in parental $(4 \mathrm{~A}+)$; Fischer et al., 2012; GSE33548]. Overexpression of IFR1 mRNA in sor1 vs. its parental strain $(4 \mathrm{~A}+)$ could be confirmed by RTqPCR experiments [median 17.8; lower quartile (Q1) 13.9; upper quartile (Q3) 25.4; IFR1 mRNA level in 4A+ set to 1; Figure 2B]. The higher mRNA level was also translated into higher IFR1 protein amounts found in sor1 (Figure 2C; 48 and $72 \mathrm{~h}$; sor1 vs. $4 \mathrm{~A}+$ ). Analysis of the IFR1 promoter region led to the identification of an 8 bp palindromic motif (CAACGTTG) (Figure 2D) which was identified as an electrophile response element (ERE) in nuclear promoters of $C$. reinhardtii genes overexpressed in the mutant sor 1 and whose expression is 

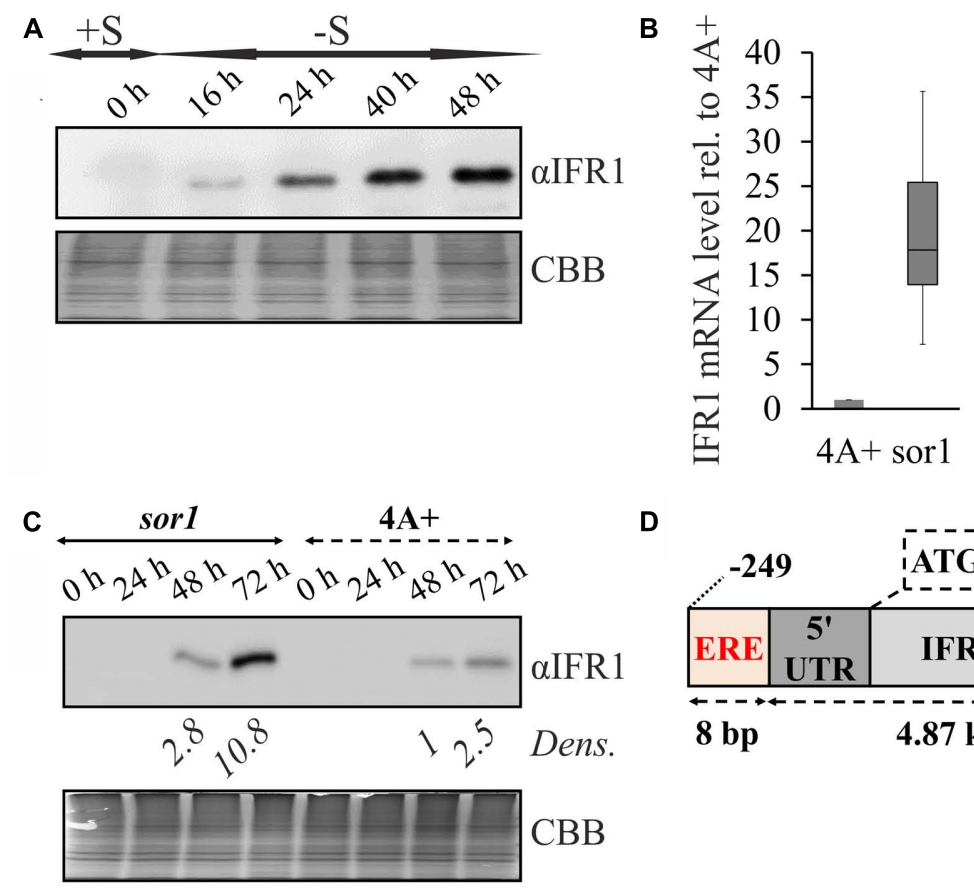

D
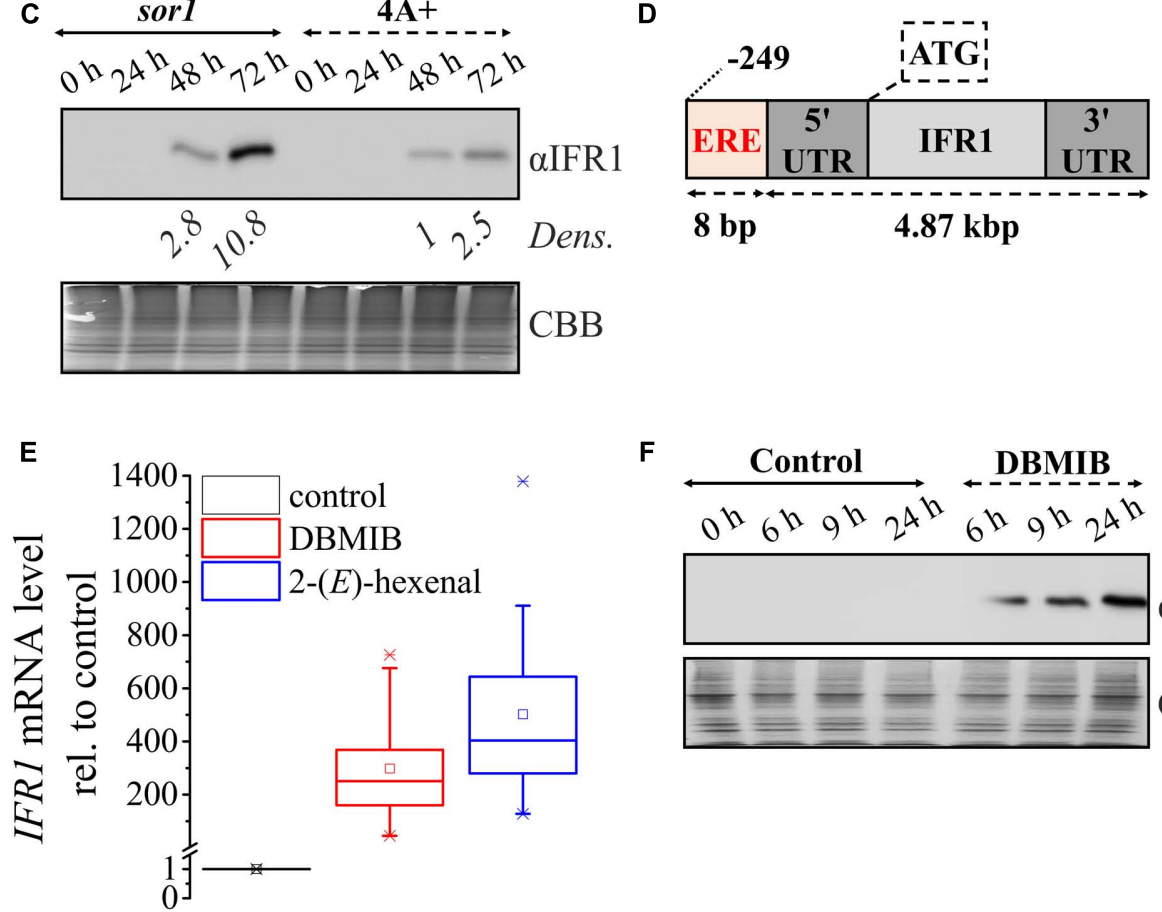

$\mathbf{F}$

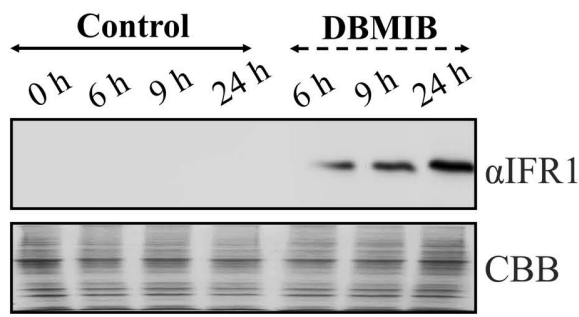

FIGURE 2 | IFR1 accumulation is triggered by the SOR1-dependent pathway. (A) Samples were taken before $(0 \mathrm{~h},+\mathrm{S})$ and during the course of hydrogen production induced by sulfur deprivation of a wild type cell line (16-48 h; -S). IFR1 accumulation was analyzed with an IFR1-specific antiserum (alFR1) and equal protein loading confirmed by colloidal Coomassie staining (CBB). (B) Comparison of IFR1 mRNA levels in the sor1 mutant (Fischer et al., 2012) and its parental strain (4A+) with samples taken in the late exponential phase. mRNA levels were determined by RTqPCR and the IFR1 transcript level in 4A+ was set to 1. Median and interquartile range shown in the box-and-whisker diagram are derived from two biological replicates, each including nine technical replicates $(n=18)$. (C) Representative immunoblot ( $\alpha$ IFR1) showing IFR1 accumulation during growth of mutant sor1 and its parental strain (4A+) in nutrient-replete TAP medium for 3 days. Relative band intensities (Dens.) determined by densitometric scanning of immunblot signals are given relative to the band intensity of the $4 \mathrm{~A}+$ sample at $\mathrm{t}_{48 \mathrm{~h}}$ (set to 1). (D) Position of the octanucleotide motif CAACGTTG described to represent an electrophile response element (ERE; Fischer et al., 2012) implicated in the genetic response to reactive electrophile species (RES) and SOR1-dependent signaling relative to the start codon (ATG) of the $4.87 \mathrm{kbp}$ IFR1 gene, comprising exons, introns and untranslated regions (UTRs). (E) IFR1 mRNA levels determined by RTAPCR following dark treatment of WT cell cultures with DBMIB $(5 \mu \mathrm{M})$ and 2-(E)-hexenal $(500 \mu \mathrm{M})$ for $24 \mathrm{~h}$. The mRNA level of the solvent control sample was set to 1. Median and interquartile range shown in the box-and-whisker diagram are derived from two biological replicates, each including six technical replicates $(n=12)$. (F) Immunoblot (alFR1) showing IFR1 accumulation distinct time points (6-24 h) after the addition of DBMIB $(5 \mu \mathrm{M})$ or only solvent (Control) to a liquid TAP culture of the $C$. reinhardtii wild type CC124 and subsequent dark incubation for $24 \mathrm{~h}$.

activated by reactive electrophile species (RES) (Fischer et al., 2012).

Indeed, treatment of $C$. reinhardtii WT cultures with the RES-compounds DBMIB (2,5-Dibromo-6-isopropyl-3-methyl1,4-benzoquinone) and 2-(E)-hexenal triggered a strong accumulation of IFR1 mRNA [median fold-induction vs. control: 250.5 for DBMIB and 403.5 for 2-(E)-hexenal; Figure 2E]. In contrast to DBMIB, 2-(E)-hexenal is a RES (oxylipin) that occurs naturally in high light-stressed cells of $C$. reinhardtii (Roach et al., 2017) and is formed from polyunsaturated fatty acids via peroxidation and subsequent enzymatic cleavage (Mosblech et al., 2009). Addition of DBMIB to sulfur-replete cultures of a C. reinhardtii $\mathrm{WT}$ in the dark induced a strong accumulation of IFR1 protein (Figure 2F). However, IFR1 protein expression 

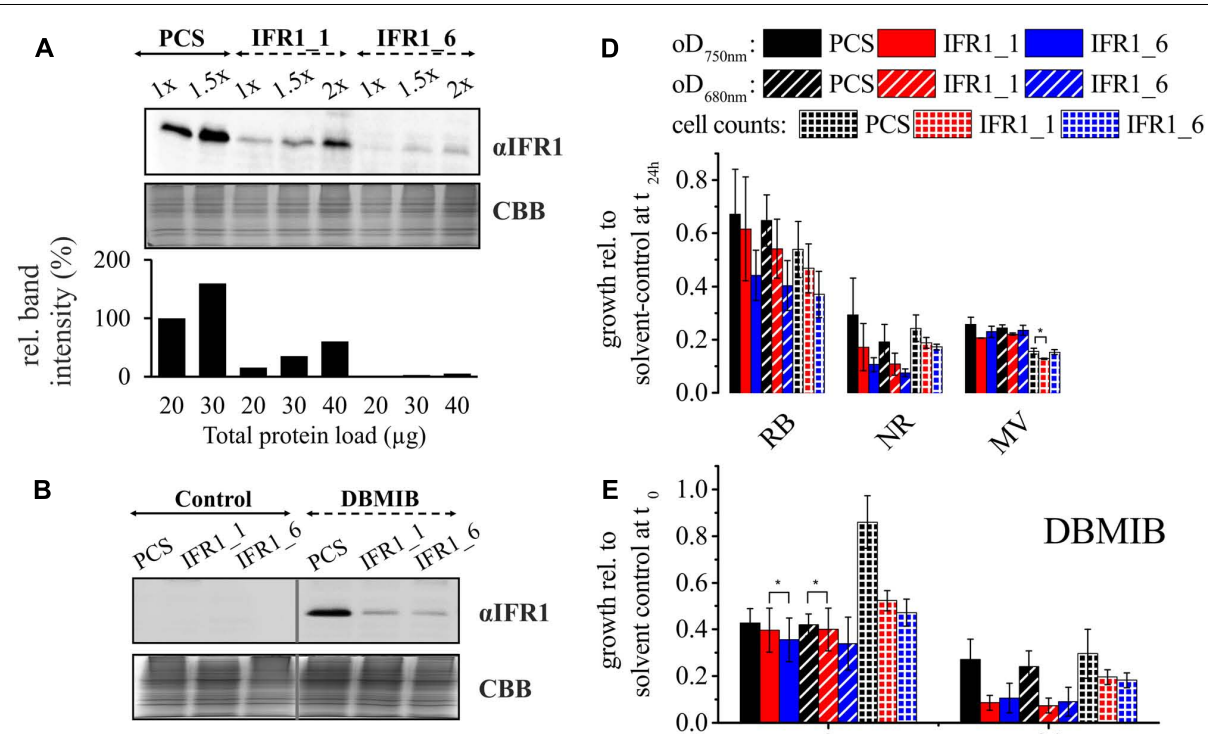

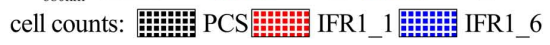
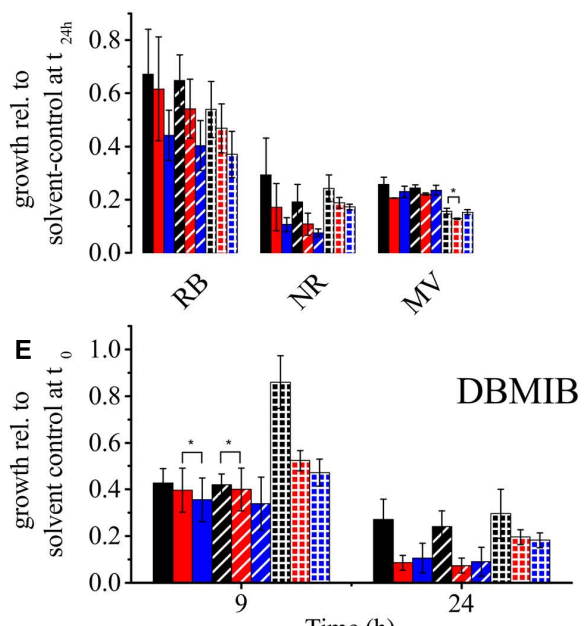

C

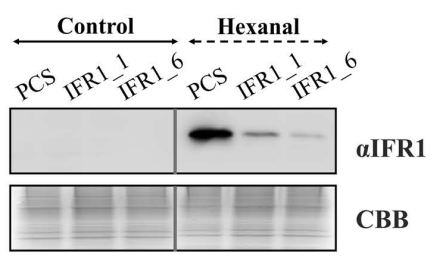

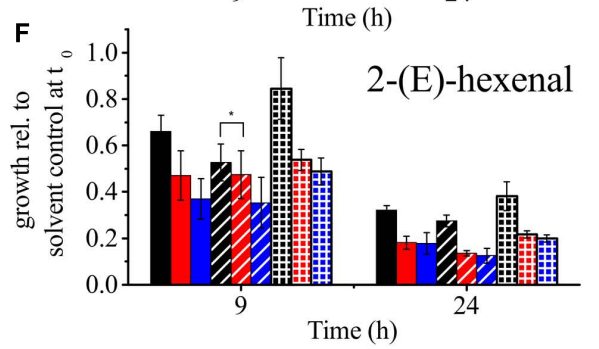

FIGURE 3 | IFR1 knock-down causes diminished tolerance toward RES in C. reinhardtii. (A) Immunodetection of IFR1 protein ( $\alpha$ IFR1) in the parental strain (PCS; wild type CC124) and IFR1 knock-down strains (IFR1_1 and IFR1_6) detected after 48 h of cultivation in sulfur deplete medium. A colloidal Coomassie stained gel (CBB) served as loading control. Different amounts of proteins were used and band intensities (lower bar diagram) determined by densitometric analysis (1x PCS set to $100 \%)$. (B,C) IFR1 accumulation in PCS and IFR1 knock-down strains grown for $24 \mathrm{~h}$ in TAP supplemented with DBMIB (5 $\mu$ M) or 2-(E)-hexenal (500 $\mu$ M). (D) Growth inhibition by reactive oxygen species determined for the PCS and the two IFR 1 knock-down strains during 24 h of growth in TAP supplemented with $4 \mu \mathrm{M}$ rose Bengal (RB), $15 \mu \mathrm{M}$ neutral red (NR), or $0.5 \mu \mathrm{M}$ methyl viologen (MV). Optical densities (determined at 680 and $750 \mathrm{~nm}$ ) and cell counts are given relative to the untreated/solvent-control sample (set to 1 ). Error bars indicate standard errors derived from three biological replicates including technical replicates $(n=3)$. Asterisks indicate significant differences between PCS and knock-down strains according to a two-tailed Student's $t$-test ( $p<0.05$ ). (E,F) Growth inhibition following treatment of PCS and IFR1 knock-down strains with $5 \mu \mathrm{M}$ DBMIB and $500 \mu \mathrm{M} 2$-(E)-hexenal for 9 or $24 \mathrm{~h}$ in TAP medium. Standard errors are derived from three biological replicates, including technical replicates $(n=3)$. Except for the data indicated by asterisks $(p>0.05)$ differences between PCS and knock-down strains were significant according to a two-tailed Student's $t$-test $(p<0.05)$.

could not be observed (data not shown) in TAP grown cultures supplemented with DCMU [3-(3,4-Dichlorophenyl)-1,1-dimethylurea; PSII forward electron inhibitor; Metz et al., 1986], indicating that inhibition of photosynthetic electron transport by DCMU or DBMIB can be excluded and noted effects can be mainly attributed to DBMIB's action as a reactive electrophile.

\section{A Knock-Down of IFR1 Causes Diminished RES-Tolerance}

To functionally characterize IFR1 of $C$. reinhardtii, we applied a reverse genetics approach, employing a nuclear expression vector for the expression of artificial microRNAs (amiRNA) (Molnar et al., 2009). Screening of transformants based on immunoblots with the IFR1-specific antiserum led to the identification of two knock-down strains. When grown in S-deplete medium, IFR1_1 and IFR1_6 accumulated $\sim 65$ and $\sim 95 \%$ less IFR1 protein, respectively as compared to the parental strain (Figure 3A).
Diminished accumulation of IFR1 in both knock-down strains was also observed, when cells were treated with the RES compound DBMIB (Figure 3B). In line with the strong IFR1 transcript accumulation observed after treatment with 2$(E)$-hexenal (Figure 2E), addition of this compound to liquid cultures triggered a strong accumulation of IFR1 protein in the parental strain, which was diminished in knock-down strains (Figure 3C). Prompted by the finding that IFR1 protein accumulates following the exposure of $C$. reinhardtii cells to RES and the reported requirement of IRL proteins for oxidative stress tolerance in higher plants (Babiychuk et al., 1995; Kim et al., 2010), we analyzed the tolerance of IFR1 knock-down strains toward various compounds which either act as reactive oxygen/electrophile species (ROS/RES) or induce their cellular accumulation (Figures 3D-F). To this end, compounds inducing ROS-stress [rose bengal (RB); neutral red (NR), and methyl viologen (MV)] or acting as 

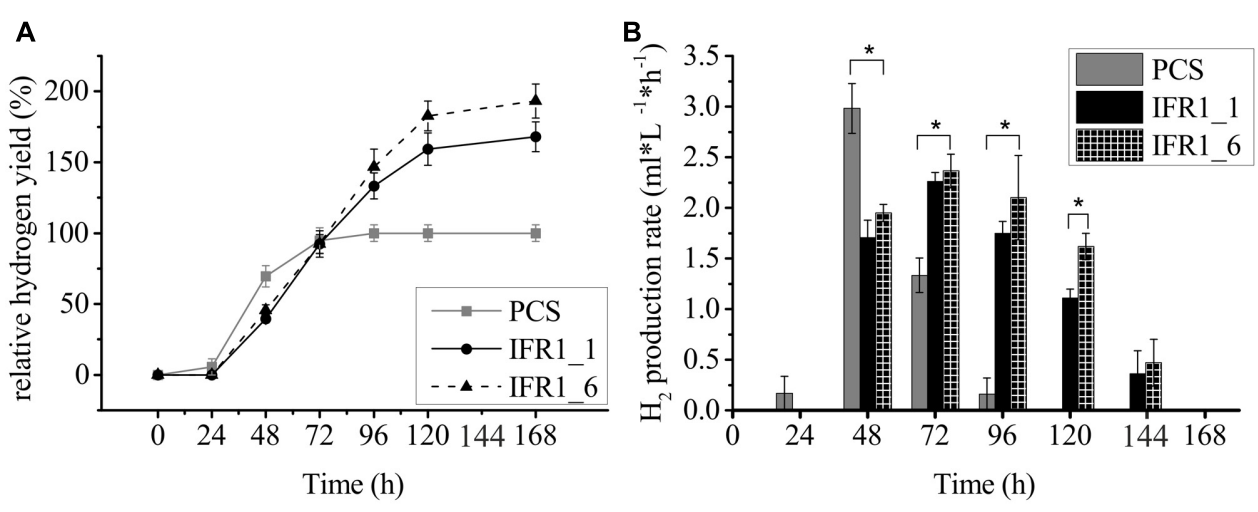

FIGURE 4 | Prolonged hydrogen production in IFR1 knock-down strains compared to the wild type. (A) Time course of hydrogen production for the parental strain (PCS) and IFR1 knock-down strains. Hydrogen yields in the knock-down strains are given relative to the final yield of the parental strain (set to 100\%). Each data curve represents an average of three biological replicates including three technical triplicates $(n=9)$ with error bars representing the standard error. (B) $\mathrm{H}_{2}$ production rates during the course of hydrogen production. Error bars indicate the standard error $(n=9)$ and asterisks indicate differences between PCS and knock-down strains which are significant according to a two-tailed Student's $t$-test $\left({ }^{*} p<0.05\right)$.

RES [DBMIB and 2-(E)-hexenal] were added to the cultures and the growth retarding-effect was quantified after $24 \mathrm{~h}$ via measurement of optical densities $\left(\mathrm{oD}_{680} \mathrm{~nm} / 750 \mathrm{~nm}\right)$ and cell densities based on cell counting. The cultures were also spotted on TAP agar plates for recovery (Supplementary Figure S3). Significant differences reflected by all growth parameters applied could not be observed regarding the susceptibility of knock-down strains vs. parental strain toward methyl viologen, which triggers superoxide formation in vivo (Babbs et al., 1989) (Figure 3D; MV). Although neutral red and rose bengal, which act as photosensitizers and trigger the formation of singlet oxygen in live cells (Fischer et al., 2004), exerted a greater growth-inhibiting effect on both knockdown strains (Figure 3D), differences between the parental and IFR1 knock-down strains were not statistically significant according to a two-tailed Student's $t$-test $(p<0.05)$. In contrast, statistically robust $(p<0.05$; two-tailed Student's $t$-test) differences could be seen when cells were treated for $24 \mathrm{~h}$ with DBMIB or 2-(E)-hexenal (Figures 3E,F), which caused a more pronounced growth inhibition in the knockdown strains. At least when cell counts were used as a growth parameter, a significantly higher susceptibility of knockdown strains toward RES could already be seen $9 \mathrm{~h}$ after the addition of DBMIB or 2-(E)-hexenal. A diminished availability of IFR1 in C. reinhardtii therefore reduces the tolerance toward RES.

\section{Prolonged Hydrogen Production by IFR1 Knock-Down Mutants}

IFR1 protein accumulates strongly in hydrogen producing cultures following sulfur-depletion (Figure 2A), indicating a potential role of this protein during the acclimation to sulfur depletion or anaerobiosis. The effect of IFR1 knock-down was assessed by measuring $\mathrm{H}_{2}$ production of the knock-down strains (Figure 4A). Parental strain, IFR1_1 and IFR1_6 were grown in sulfur-replete TAP medium to a mid-log phase and transferred to sulfur-deplete TAP medium by adjusting them to the same starting chlorophyll concentration $(\sim 25 \mu \mathrm{g} / \mathrm{ml})$. Hydrogen production was first notable $48 \mathrm{~h}$ after the onset of sulfur depletion and at the beginning hydrogen yields in the PCS exceeded those of the knock-down strains by $\sim 35-$ $40 \%$. During the course of $\mathrm{H}_{2}$ production, production rates declined in the PCS from $48 \mathrm{~h}$ onward, while rates in the knock-down strains increased toward $\mathrm{t}_{72} \mathrm{~h}$ and started declining notably beyond the time point $\mathrm{t}_{120 \mathrm{~h}}$ (Figure 4B). The $\mathrm{H}_{2}$ production phase in PCS ceased at $96 \mathrm{~h}$ with a production phase (time between the first detection and the end of $\mathrm{H}_{2}$ production) of 3 days as compared to 5 days by IFR 1 knockdown strains. Although the highest rate of hydrogen production $\left(2.98 \pm 0.25 \mathrm{ml} \mathrm{L}^{-1} \mathrm{~h}^{-1}\right)$ was reached in the PCS strain, the prolonged hydrogen production in the knock-down strains eventually led to final hydrogen yields that were about $68 \pm 10 \%$ (SE) (IFR1-1) and $93 \pm 12 \%$ (IFR1-6) higher than the yield from the wild type.

\section{Prolonged $\mathrm{H}_{2}$ Production in IFR1 Knock-Down Strains Results from a Sustained PSII Activity}

One of the reasons for a prolonged hydrogen production in the course of sulfur starvation, could be a high residual PSII activity, which is required for efficient hydrogen production (Volgusheva et al., 2013; Steinbeck et al., 2015). Indeed, both IFR1 knock-down strains displayed a higher residual activity of PSII, measured as the maximum quantum yield of dark-adapted cells $\left(\mathrm{F}_{v} / \mathrm{F}_{m}\right)$, following the exposure to sulfur limitation under aerobic conditions (Figure $\mathbf{5 A} ;-\mathrm{S} /+\mathrm{O}_{2}$; $\mathrm{Fv} / \mathrm{Fm} ; \mathrm{t}_{72-168 \mathrm{~h}}$ ). The knock-down strain IFR1_6 was then selected for more detailed analyses regarding differences in PSII stability between knock-down and parental strain under hydrogen production conditions (sulfur deprivation under anaerobic conditions). Also during the course of hydrogen production, the knock-down of IFR1 causes an 

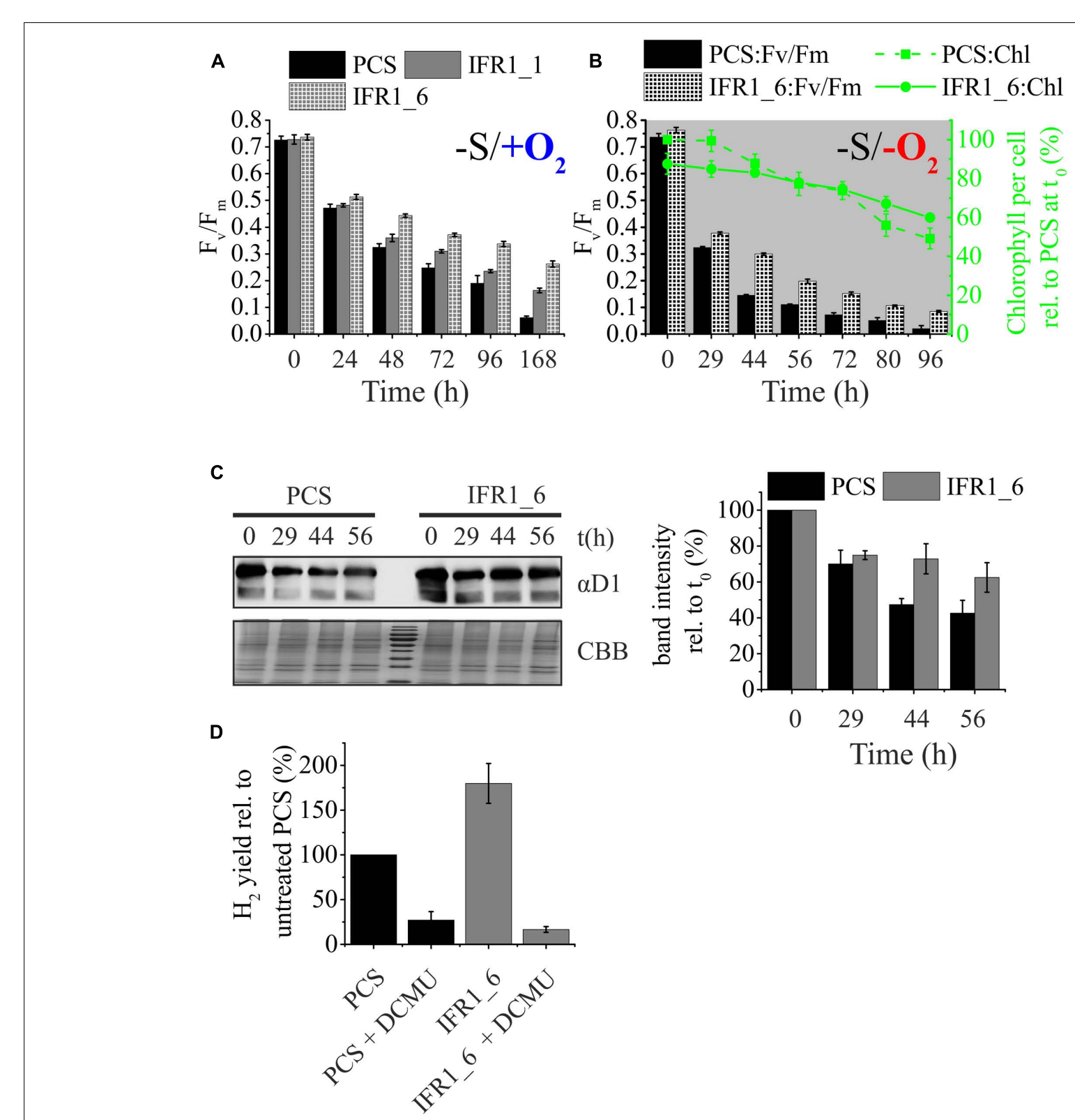

FIGURE 5 | Contribution of PSII and photosynthetic/respiration $(P / R)$ rates on hydrogen production. (A) Maximum quantum yield $\left(F_{v} / F_{m}\right)$ of dark-adapted cells of the parental strain (PCS) and IFR1 knock-down strains (IFR1_1/IFR1_6) before ( $\left.t_{0}\right)$ and after exposure to sulfur limitation ( $t_{24}-t_{168} \mathrm{~h}$ ) and aerobic conditions. Error bars indicate the standard error from three biological replicates $(n=3)$. (B) Time course of the maximum quantum yield $\left(F_{v} / F_{m}\right.$; left $y$-axis) and the cellular chlorophyll content (right y-axis) during photosynthetic hydrogen production of the parental strain (PCS) and one of the IFR1 knock-down strains (IFR1_6). Chlorophyll data were normalized to the chlorophyll content of PCS at to (set to 100\%). Standard errors derived from three biological replicates $(n=3)$ are indicated as error bars. Except for $t_{0}$, the differences between PCS and IFR1_6 in regard to $F_{v} / F_{m}$ were significant according to a two-tailed Student's $t$-test $(p<0.05)$. (C) Representative immunoblot showing the immunodetection of PSII subunit D1 (upper left panel; $\alpha$ D1) in samples of the parental strain (PCS) and IFR1_6 taken at indicated times during a hydrogen production experiment. A colloidal Coomassie stain (lower left panel; CBB) served as a loading control. Results from densitometric scanning (right panel) of blot signals are given relative to the D1 signal intensity determined for $t_{0}$ (set to $100 \%$ ). Error bars indicate standard errors (three biological replicates; $n=3$ ). (D) Relative $\mathrm{H}_{2}$ yields obtained with the parental control strain (PCS) (black bars) and knock-down strain IFR1_6 (gray bars) in the absence or presence of $20 \mu \mathrm{M}$ DCMU. Hydrogen yields determined for the untreated PCS were set to 100\%. Error bars represent standard error $(n=6)$.

increased stability of PSII, as seen by higher $\mathrm{F}_{v} / \mathrm{F}_{m}$ values from time point $t_{29}$ onward (Figure $5 \mathrm{~B} ;-\mathrm{S} /-\mathrm{O}_{2}$; left y-axis; PCS vs. IFR1_6). The lowered susceptibility of PSII toward photoinhibition in the IFR1_6 was also reflected by a lower relative decrease in the cellular chlorophyll content
( $\sim 30 \%$ vs. $50 \%$ in PCS; Figure 5B; right y-axis; green curves). In good agreement with the higher residual PSII activity found in IFR1 knock-down strains (Figures 5A,B), the cellular content of the PSII core subunit D1 declined more slowly within the course of hydrogen production in 
knock-down strain IFR1_6 compared to its parental strain (Figure 5C).

Specific inhibition of PSII with DCMU was used to confirm if the electrons for prolonged $\mathrm{H}_{2}$ production indeed originated from residual PSII activity. DCMU was added directly into the $\mathrm{H}_{2}$ bioreactors $30 \mathrm{~h}$ after the onset of sulfur deprivation. DCMU blocks the PSII-dependent pathway of hydrogen production based on residual water-splitting activity and linear electron transport toward the hydrogenase enzyme and inhibits $\mathrm{H}_{2}$ production substantially, as reported before (Kruse et al., 2005; Volgusheva et al., 2007; Scoma et al., 2014). $\mathrm{H}_{2}$ production dropped in both strains upon addition of DCMU (Figure 5D), but the relative effect of DCMU on hydrogen production was much stronger in the IFR1 knock-down strain (73\% reduction in PCS vs. $163 \%$ reduction in IFR1_6). Furthermore total hydrogen production in strain IFR1_6 (16.6 $\pm 3.2 \%)$ was lower than the production observed for the parental strain $(27.1 \pm 9.4 \%)$, when DCMU was added to inhibit PSII. It can thus be concluded that the increased hydrogen production capacity caused by a knock-down of IFR1 mainly results from an enhanced activity of the PSII-dependent pathway, especially during the later stages of the hydrogen production pathway, when PSII activity in IFR1 knock-down strains exceeds the respective activity seen in the parental strain (Figures 5A-C).

\section{IFR1 Knock-Down Can Be Applied as a Tool to Further Enhance Hydrogen Production in a Strain with a High Starting Capacity}

To test whether the knock-down of IFR1 can be applied as a tool to improve the hydrogen production capacity in various C. reinhardtii strains, we selected the strain stm6, known to produce high amounts of hydrogen (Kruse et al., 2005; Doebbe et al., 2010; Nguyen et al., 2011). One of the created IFR1 knockdown strains, stm6_IFR $1 k d$, displayed an IFR1 accumulation diminished to $\sim 20 \%$ of the IFR1 level found in the parental strain stm6 (Figure 6A; $\alpha$ IFR1; $1 \mathrm{X}$ stm6 vs. 1X stm6_IFRkd). Confirming the results obtained with knock-down strains derived from a wild type cell line (Figure 4), an IFR1 knock-down in the background of strain stm6 also had a tremendous impact on the time course of hydrogen production and the overall production capacity (Figure 6B). The onset of hydrogen production in strain stm6_IFRkd (Figure 6B; gray curve) was delayed by $\sim 20 \mathrm{~h}$ compared to the parental strain (black curve), but hydrogen production in the knock-down strain reached a plateau phase only at time point $168 \mathrm{~h}$, while the parental strain reached this phase already before $t_{120} \mathrm{~h}$. A prolonged hydrogen production phase together with an increased $\mathrm{H}_{2}$ productivity rate (up to $3.07 \mathrm{ml} \cdot \mathrm{L}^{-1} \cdot \mathrm{h}^{-1}$ at $\mathrm{t}_{72 \mathrm{~h}}$ ), indicated by a steeper slope of the stm6_IFRkd curve, resulted in a final hydrogen yield of the knock-down strain which was $70 \%$ higher than the respective yield obtained with the parental strain.

In analogy to what has been observed for the IFR1 knockdown strains derived from a wild type, PSII activity $\left(\mathrm{F}_{v} / \mathrm{F}_{m}\right.$;
A
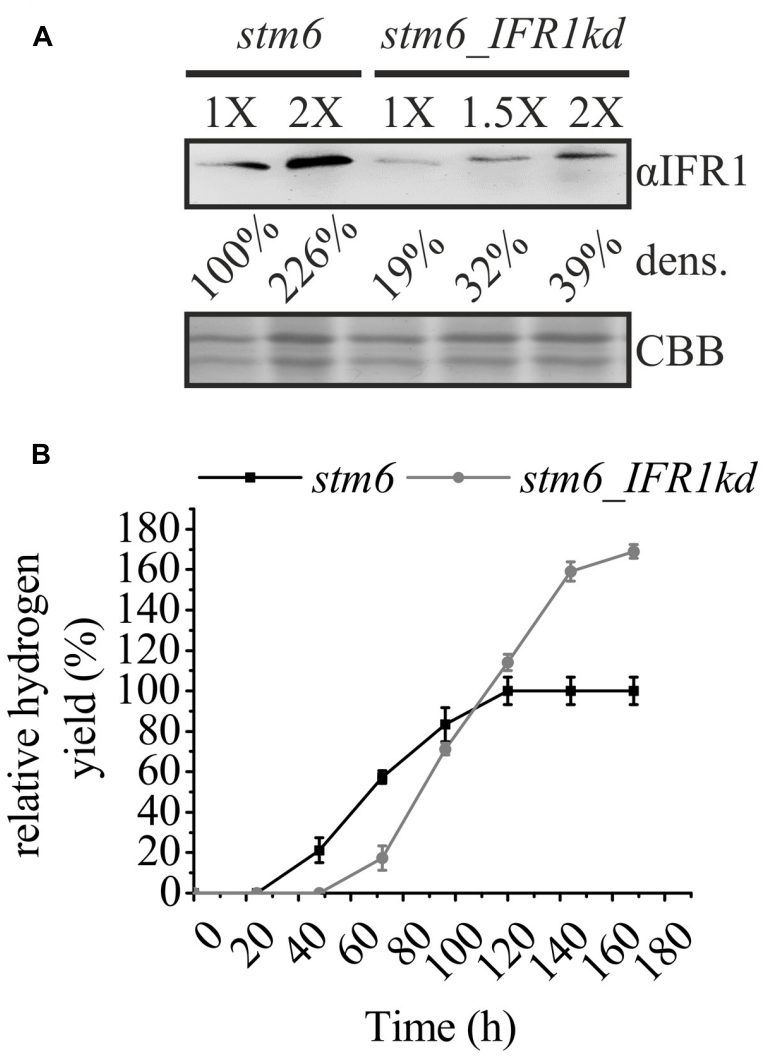

C

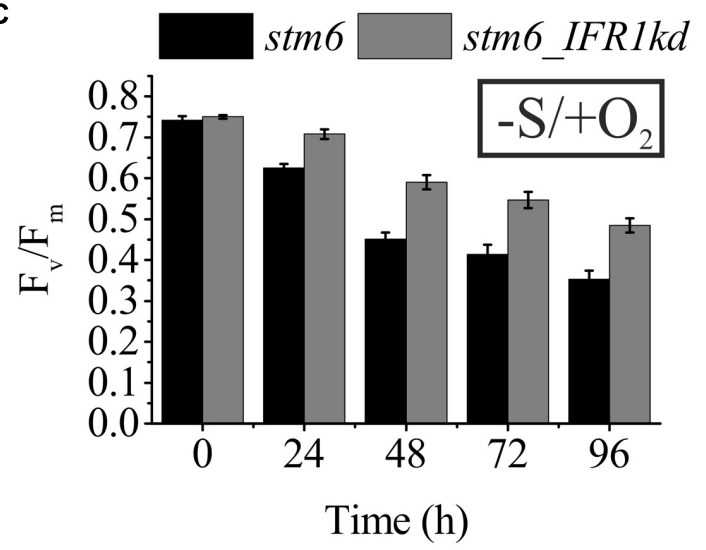

FIGURE 6 | Knock-down of IFR1 in the boosts hydrogen production in the high hydrogen producer mutant stm6. (A) Immunoblot analysis of IFR1 accumulation in stm6 and stm6_IFR1 $k d$ cultivated under sulfur-limiting conditions. Different amounts of total protein (1X; 1.5X; and 2X) were used for immunodetection of IFR1 ( $\alpha$ IFR1) with a colloidal Coomassie stain (CBB) serving as a loading control. Results from densitometric signal analysis (dens.) are indicated. (B) Relative time-dependent $\mathrm{H}_{2}$ yields of the stm6 parental strain (black curve) and stm6_IFR1 kd (gray curve) with the final yield in stm6 set to $100 \%$. Error bars represent the standard error (three biological replicates including technical triplicates, $n=9$ ). (C) Maximum quantum yield of PSII determined after dark incubation ( $\mathrm{Fv} / \mathrm{Fm}$ ) determined in cultures of stm6 (black bars) and stm6_IFR1kd (gray bars) exposed to sulfur starvation. Standard errors, shown as error bars are derived from three biological replicates including technical duplicates $(n=6)$. Except for to, differences between stm6 and stm6_IFR1 $\mathrm{kd}$ were significant according to a two-tailed Student's $t$-test $(p<0.05)$. 
Figure 6C) declined more slowly in stm6_IFRkd vs. stm6 when cells were cultivated under aerobic sulfur-limiting conditions $\left(0.48 \pm 0.01\right.$ in stm6_IFRkd vs. $0.35 \pm 0.02$ in stm6 at $\left.t_{96 h}\right)$. In summary, these results demonstrate again that a diminished IFR1 level boosts hydrogen production in C. reinhardtii and that this effect is based on a sustained residual PSII activity which extends the hydrogen production phase significantly. The correlation between cellular amounts of IFR1 and hydrogen production capacity is further underscored by the diminished hydrogen yields obtained with the sor 1 mutant (Supplementary Figure S4), which overexpresses IFR1 (Figures 2B,C), in comparison to its parental strain $(4 \mathrm{~A}+)$.

\section{DISCUSSION}

In silico analyses performed with the amino acid sequence of IFR1 revealed that this protein represents an atypical member of the short-chain dehydrogenase/reductase (SDR) superfamily. Several SDRs including IFR1 from $C$. reinhardtii have been suggested to be referred to as NmrA-like family proteins (family designation SDR48A) according to a nomenclature initiative of Persson et al. (2009). Most of these SDRs including IFR1, however, share significantly higher percent identities with isoflavone reductases (Arabidopsis thaliana IFR; 29\% identity to IFR1), IRL proteins (Zea mays IRL; $\sim 29 \%$ ), phenylcoumaran benzylic ether reductases (PCBER; $\sim 28 \%$ ) or eugenol synthases (EGS; $24 \%$ ) from higher plants than with the $\mathrm{N}$ metabolite repression protein $\mathrm{A}$ (NmrA; 20\%) from Aspergillus (Emericella) nidulans (Supplementary Figure S1 and Table S1). A wide-scale bioinformatics study on SDRs in plant genomes suggested a distinct SDR family for IFR, PCBER and eugenol synthase (family designation SDR460A) and although IFR1 was 1 of $15 \mathrm{C}$. reinhardtii proteins that could not be assigned to any SDR family during that study, a high homology of IFR1 to members of the SDR460A family was claimed, however (Moummou et al., 2012).

In the legume alfalfa (Medicago sativa), isoflavone reductase (IFR) catalyzes the stereospecific reduction of $2^{\prime}$ hydroxyformononetin to yield (3R)-vestitone (Dewick, 1977; Paiva et al., 1991) as part of the biosynthesis pathway for the isoflavonoid (-)-medicarpin (Guo and Paiva, 1995). Isoflavonoids and IFRs are almost entirely confined to legumes and, although flavonoids have been identified as sex pheromones in Chlamydomonas eugamentos (Birch et al., 1953), no isoflavonoids or IFRs have been reported in C. reinhardtii (May et al., 2008; Annamalai and Nallamuthu, 2014). Several IFR-like (IRL) proteins have been cloned from non-leguminous plants (Babiychuk et al., 1995; Petrucco et al., 1996; Shoji et al., 2002; Kim et al., 2003; Hua et al., 2013). Despite their high homology toward IFR proteins, IRL proteins do not accept $2^{\prime}$-hydroxyformononetin as a substrate (Petrucco et al., 1996) and for several higher plant IRLs their specific induction by abiotic stresses such as exposure to reactive oxygen species (Kim et al., 2010) or UV light (Lers et al., 1998) has been demonstrated. For the IRL proteins from maize and rice, a strong negative correlation between cellular glutathione (GSH) levels and the expression level of IRLs was shown (Petrucco et al., 1996). Sulfur depletion, which triggers the accumulation of maize IRL, is a condition known to cause a strong decline of GSH levels in green algae (Salbitani et al., 2015) and higher plants (Kopriva and Rennenberg, 2004). As observed within the present study, C. reinhardtii IFR1 also accumulates upon sulfur limitation, whereas its expression is low under stress-free conditions (Figure 2A).

In further analogy to IRL proteins from maize and rice, expression of the $C$. reinhardtii IFR1 gene is also induced by reactive oxygen species such as hydrogen peroxide (Blaby et al., 2015). Molecular details on the expression regulation of higher plant $I R L$ genes have thus far remained obscure, whereas for IFR1 the present study provides strong evidence for the function of an ERE (Fischer et al., 2012) as a cis-regulatory IFR1 promoter sequence required for the accumulation of IFR1 following exposure to reactive electrophiles (Figures 2B-F, 3C). The ERE cis-regulatory element (CAACGTTG) was identified as a palindromic sequence overrepresented in the -70 to -340 bp promoter region of genes overexpressed in the singlet oxygen resistant 1 (sor 1) mutant and the ERE of IFR1 lies within this region ( $-249 \mathrm{bp}$; Figure 2D). It was shown to be required for the induction and overexpression of ROS/RES-defense genes (glutathione-S-transferase 1; GSTS1) by the lipophilic REScompound DBMIB in mutant sor1. Reporter constructs containing the ERE responded more strongly and much faster to lipophilic RES than to hydrophilic chemicals producing ROS. From this and other results it was therefore concluded that ERE activation via ROS is indirect and based on lipid peroxidation triggered by ROS and generating lipophilic RES such as malondialdehyde (Fischer et al., 2012). Among a variety of ROS- and RES-generating chemicals tested, GSTS1 reporter constructs containing ERE elements responded most strongly to DBMIB and 2-(E)-hexenal (Fischer et al., 2012), and IFR1 transcript (Figure 2E) and protein (Figures 2F, 3B,C) accumulates upon treatment of C. reinhardtii cells with these compounds. Furthermore, IFR 1 mRNA and protein over-accumulate in the sor 1 mutant (Figures 2B,C).

In addition to their strong effects on IFR1 expression, the growth-retarding effect of DBMIB and 2-(E)-hexenal is more prominent in IFR1 knock-down strains than in their parental strain (Figures 3E,F). It is therefore tempting to speculate, that the short-chain dehydrogenase/reductase (SDR) IFR1 might be involved in the detoxification of these compounds, since (SDRs) have already been shown to act as cytosolic aldehyde reductases (CytADRs) in A. thaliana (Yamauchi et al., 2011). In principle, highly reactive 2 -alkenals from lipid peroxidation can be detoxified either by reduction of the aldehyde group or by reduction of the $\alpha, \beta$-unsaturated bond (Mano et al., 2005; Yamauchi et al., 2011). CytADRs catalyze the latter reaction and represent typical SDRs while IFR1 is an atypical SDR (aSDR) according to its $\mathrm{NAD}(\mathrm{P}) \mathrm{H}$ binding motif of the G-X-X-G-X$\mathrm{X}-\mathrm{G}$ type (Supplementary Figure S1). Alignment of the IFR1 
amino sequence with those of CytADRs from $A$. thaliana revealed percent identities in the range of $19-21 \%$, whereas a C. reinhardtii SDR (Cre12.g549852) whose expression is induced by 2-(E)hexenal (Fischer et al., 2012) shows a higher degree of identity to CytADRs (23-25\% identity; Supplementary Table S1). In A. thalinana, enzymes reducing reactive carbonyls have been identified within the medium-chain dehydrogenase/reductase (MDR) superfamily, NADB_Rossmann (SDR) superfamily and aldo-keto reductase (AKR) superfamily (Yamauchi et al., 2011), but aSDRs implicated in the detoxification of reactive carbonyls have not been identified so far. In vitro assays based on NADPH consumption monitored via absorbance change at $340 \mathrm{~nm}$ with recombinant IFR1 and 2-(E)-hexenal (data not shown) did not indicate that this compound could represent a substrate in vivo.

Apart from the treatment of cell cultures with lipophilic RES, sulfur deprivation is a condition that is associated with IFR1 accumulation (Figure 2A). In microalgae sulfur limitation is known to cause the formation of reactive oxygen species (Salbitani et al., 2015), which could in turn trigger lipid peroxidation resulting in the production of reactive carbonyls/RES (Mosblech et al., 2009; Roach et al., 2017). At the same time, the withdrawal of sulfur diminishes the glutathione pool size (Salbitani et al., 2015) and a large pool of reduced glutathione (GSH) protects cellular components (e.g., amino groups from DNA bases or within proteins) against modification by RES via scavenging as GSH-conjugates and subsequent detoxification (Mueller and Berger, 2009). It is therefore possible that IFR1 induction under sulfur-deplete conditions proceeds via the accumulation of RES, originating from $-S$-triggered ROS formation and simultaneous impairment of GSH-dependent scavenging, and an activation of the SOR1-dependent pathway. This could also provide an explanation for the finding that nitrogen limitation does not induce IFR1 accumulation, because at least in higher plants, effects of nitrogen deficiency on foliar GSH levels are rather small compared to those exerted by sulfur withdrawal (Koprivova et al., 2000).

Although IFR1 does not seem to be involved in the direct detoxification of RES, a diminished amount of IFR1 reduces RES tolerance in C. reinhardtii (Figures 3E,F), indicating that IFR1 is somehow implicated in the regulation of RES homeostasis. The precise in vivo function of IFR1 within the context of RES homeostasis in C. reinhardtii can, however, not be depicted at the moment. Since in vivo substrate identification based on in silico analyses of SDRs and aSDRs is not feasible, future research on IFR1 will have to comprise the cumbersome screening of compound libraries (Bhatia et al., 2015).

Intriguingly, a knock-down of IFR1 in C. reinhardtii boosts hydrogen production (Figures 4, 6). In C. reinhardtii, the production of hydrogen can be triggered by sulfur deprivation in air-tight cultures (Melis et al., 2000). A strong downregulation of the Calvin-Benson cycle is thought to over-reduce the photosynthetic electron transport chain, a condition which promotes the formation of ROS. ROS damage the photosynthetic apparatus, especially photosystem II, and a diminished activity of the PSII repair cycle in the absence of sulfur further contributes to a strong decline in PSII activity, which in turn gradually decreases the oxygen content of cultures, because mitochondrial respiration is less affected by sulfur deprivation (Ghysels and Franck, 2010). A strong decline of PSII activity could also be noted under sulfur limitation in the present study (Figures 5A,B, 6C) and remarkable differences were seen between parental strains and IFR1 knock-down mutants. Interestingly, in strains expressing lower amounts of IFR1, PSII was less susceptible to $-\mathrm{S}$-induced photoinhibition, as could be seen as higher $\mathrm{F}_{v} / \mathrm{F}_{m}$ values (Figures 5A,B, 6C) and a slower decline in the levels of D1 protein (Figure 5C), constituting the PSII core complex. The increased stability of PSII in knock-down strains caused a prolonged hydrogen production phase in strains with diminished IFR1 levels. Therefore, the data from the present study nicely underscore the importance of residual water-splitting activity for -S-induced hydrogen production, which was also observed in previous studies (Volgusheva et al., 2013).

The simultaneous occurrence of a higher RES sensitivity and increased stability of PSII as important phenotypic characteristics of IFR1 knock-strains first seems counterintuitive. In this context, however, it must be emphasized that RES should not be merely viewed as cytotoxic compounds that need be rapidly removed from the cellular metabolism in order to prevent cell damage. Numerous studies indicate a role of RES as important signaling molecules which represent a central component of abiotic stress responses (Yamauchi et al., 2015; Muench et al., 2016). For instance, 2-(E)-hexenal has been shown to be a strong inducer of genetic programs activated as part of abiotic stress responses (Copolovici et al., 2012; Yamauchi et al., 2015) and this compound is formed under photooxidative stress conditions [e.g., as experienced by plants devoid of non-photochemical quenching mechanisms (Loreto et al., 2006)]. The PSII-damaging effect of 2-(E)-hexenal was found to be rather subtle in higher plants (Yamauchi et al., 2015). Therefore, the more pronounced growth retarding effect of 2(E)-hexenal seen for IFR1 knock-down strains might be based on mechanisms other than PSII inhibition. Overall, it seems feasible that perturbation of RES-dependent signaling could also result in a higher stress tolerance of cells (e.g., by a diminished threshold for the activation of stress response mechanisms or even their constitutive activation).

As a novel finding, manipulation of RES homeostasis in C. reinhardtii can be used to increase photosynthetic hydrogen production. Although the precise molecular function of IFR1 in Chlamydomonas is difficult to depict at the moment, the regulation of IFR1 expression via a characterized ERE, its strong over-accumulation in the sor 1 mutant and the lowered RES tolerance indicate that IFR1 is a factor required for RES-dependent signaling or RES handling in this microalga (Figures 2B-D, 3E,F). IFR1 will thus represent an important tool for future studies regarding the role of RES in abiotic stress responses of $C$. reinhardtii.

\section{AUTHOR CONTRIBUTIONS}

DV performed most of the experiments; DV, SH, CS, and TB performed and designed experiments; DV, LW, AP, and OK 
conceived the project and wrote the article with contributions of all the authors.

\section{FUNDING}

This work is supported by the Ministry of Innovation, Science, Research and Technology of the State of North Rhine-Westphalia (MIWFT) as part of the research cooperation "MoRitS Model-based Realization of intelligent Systems in Nano- and Biotechnologies" (grant no. 321 - 8.03.04.03 - 2012/02).

\section{REFERENCES}

Andrianopoulos, A., Kourambas, S., Sharp, J. A., Davis, M. A., and Hynes, M. J. (1998). Characterization of the Aspergillus nidulans nmra gene involved in nitrogen metabolite repression. J. Bacteriol. 180, 1973-1977.

Annamalai, J., and Nallamuthu, T. (2014). Antioxidant potential phytochemicals from methanol extract of Chlorella vulgaris and Chlamydomonas reinhardtii. J. Algal Biomass Util. 5, 60-67.

Antal, T. K., Krendeleva, T. E., Laurinavichene, T. V., Makarova, V. V., Ghirardi, M. L., Rubin, A. B., et al. (2003). The dependence of algal $\mathrm{H}_{2}$ production on Photosystem II and $\mathrm{O}_{2}$ consumption activities in sulfur-deprived Chlamydomonas reinhardtii cells. Biochim. Biophys. Acta 1607, 153-160. doi: 10.1016/j.bbabio.2003.09.008

Babbs, C. F., Pham, J. A., and Coolbaugh, R. C. (1989). Lethal hydroxyl radical production in paraquat-treated plants. Plant Physiol. 90, 1267-1270. doi: 10. 1104/pp.90.4.1267

Babiychuk, E., Kushnir, S., Belles-Boix, E., Van Montagu, M., and Inze, D. (1995). Arabidopsis thaliana NADPH oxidoreductase homologs confer tolerance of yeasts toward the thiol-oxidizing drug diamide. J. Biol. Chem. 270, 2622426231. doi: 10.1074/jbc.270.44.26224

Baltz, A., Dang, K.-V., Beyly, A., Auroy, P., Richaud, P., Cournac, L., et al. (2014). Plastidial expression of type II NAD(P)H dehydrogenase increases the reducing state of plastoquinones and hydrogen photoproduction rate by the indirect pathway in Chlamydomonas reinhardtii. Plant Physiol. 165, 1344-1352. doi: 10.1104/pp.114.240432

Bhatia, C., Oerum, S., Bray, J., Kavanagh, K. L., Shafqat, N., Yue, W., et al. (2015). Towards a systematic analysis of human short-chain dehydrogenases/reductases (SDR): ligand identification and structure-activity relationships. Chem. Biol. Interact. 234, 114-125. doi: 10.1016/j.cbi.2014.12.013

Birch, A. J., Donovan, F. W., and Moewus, F. (1953). Biogenesis of flavonoids in Chlamydomonas eugametos. Nature 172, 902-904. doi: 10.1038/172902a0

Blaby, I. K., Blaby-Haas, C. E., Perez-Perez, M. E., Schmollinger, S., Fitz-Gibbon, S., Lemaire, S. D., et al. (2015). Genome-wide analysis on Chlamydomonas reinhardtii reveals the impact of hydrogen peroxide on protein stress responses and overlap with other stress transcriptomes. Plant J. 84, 974-988. doi: 10.1111/ tpj.13053

Chochois, V., Dauvillee, D., Beyly, A., Tolleter, D., Cuine, S., Timpano, H., et al. (2009). Hydrogen production in Chlamydomonas: photosystem II-dependent and -independent pathways differ in their requirement for starch metabolism. Plant Physiol. 151, 631-640. doi: 10.1104/pp.109.144576

Copolovici, L., Kannaste, A., Pazouki, L., and Niinemets, U. (2012). Emissions of green leaf volatiles and terpenoids from Solanum lycopersicum are quantitatively related to the severity of cold and heat shock treatments. J. Plant Physiol. 169, 664-672. doi: 10.1016/j.jplph.2011.12.019

Debuchy, R., Purton, S., and Rochaix, J. D. (1989). The argininosuccinate lyase gene of Chlamydomonas reinhardtii: an important tool for nuclear transformation and for correlating the genetic and molecular maps of the ARG7 locus. EMBO J. 8, 2803-2809.

Dewick, P. M. (1977). Biosynthesis of pterocarpan phytoalexins in Trifolium pratense. Phytochemistry 16, 93-97. doi: 10.1016/0031-9422(77)83020-3

Doebbe, A., Keck, M., La Russa, M., Mussgnug, J. H., Hankamer, B., Tekçe, E., et al. (2010). The interplay of proton, electron, and metabolite supply for

\section{ACKNOWLEDGMENT}

We are grateful to the Center for Biotechnology (CeBiTec) at Bielefeld University for access to the Technology Platforms.

\section{SUPPLEMENTARY MATERIAL}

The Supplementary Material for this article can be found online at: http://journal.frontiersin.org/article/10.3389/fpls.2017.01347/ full\#supplementary-material

photosynthetic $\mathrm{H}(2)$ production in Chlamydomonas reinhardtii. J. Biol. Chem. 285, 30247-30260. doi: 10.1074/jbc.M110.122812

Doebbe, A., Rupprecht, J., Beckmann, J., Mussgnug, J. H., Hallmann, A., Hankamer, B., et al. (2007). Functional integration of the HUP1 hexose symporter gene into the genome of C. reinhardtii: impacts on biological $\mathrm{H}_{2}$ production. J. Biotechnol. 131, 27-33. doi: 10.1016/j.jbiotec.2007.05.017

Dubini, A., and Ghirardi, M. L. (2015). Engineering photosynthetic organisms for the production of biohydrogen. Photosynth. Res. 123, 241-253. doi: 10.1007/ s11120-014-9991-x

Fang, W., Si, Y., Douglass, S., Casero, D., Merchant, S. S., Pellegrini, M., et al. (2012). Transcriptome-wide changes in Chlamydomonas reinhardtii gene expression regulated by carbon dioxide and the $\mathrm{CO}_{2}$-concentrating mechanism regulator CIA5/CCM1. Plant Cell 24, 1876-1893. doi: 10.1105/tpc.112.097949

Filling, C., Berndt, K. D., Benach, J., Knapp, S., Prozorovski, T., Nordling, E., et al. (2002). Critical residues for structure and catalysis in short-chain dehydrogenases/reductases. J. Biol. Chem. 277, 25677-25684. doi: 10.1074/jbc. M202160200

Fischer, B. B., Krieger-Liszkay, A., and Eggen, R. I. L. (2004). Photosensitizers neutral red (Type I) and rose bengal (Type II) cause light-dependent toxicity in Chlamydomonas reinhardtii and induce the Gpxh gene via increased singlet oxygen formation. Environ. Sci. Technol. 38, 6307-6313. doi: 10.1021/es049673y

Fischer, B. B., Ledford, H. K., Wakao, S., Huang, S. G., Casero, D., Pellegrini, M., et al. (2012). SINGLET OXYGEN RESISTANT 1 links reactive electrophile signaling to singlet oxygen acclimation in Chlamydomonas reinhardtii. Proc. Natl. Acad. Sci. U.S.A. 109, E1302-E1311. doi: 10.1073/pnas.1116843109

Gaffron, H., and Rubin, J. (1942). Fermentative and photochemical production of hydrogen in algae. J. Gen. Physiol. 26, 219-240. doi: 10.1085/jgp.26.2.219

Ghirardi, M. L., Togasaki, R. K., and Seibert, M. (1997). Oxygen sensitivity of algal $\mathrm{H}_{2}$-production. Appl. Biochem. Biotechnol. 6, 141-151. doi: 10.1007/ BF02920420

Ghysels, B., and Franck, F. (2010). Hydrogen photo-evolution upon S deprivation stepwise: an illustration of microalgal photosynthetic and metabolic flexibility and a step stone for future biotechnological methods of renewable $\mathrm{H}_{2}$ production. Photosynth. Res. 106, 145-154. doi: 10.1007/s11120-010-9582-4

González-Ballester, D., Casero, D., Cokus, S., Pellegrini, M., Merchant, S. S., and Grossman, A. R. (2010). RNA-seq analysis of sulfur-deprived Chlamydomonas cells reveals aspects of acclimation critical for cell survival. Plant Cell 22, 2058-2084. doi: 10.1105/tpc.109.071167

Grossman, A. R., Catalanotti, C., Yang, W., Dubini, A., Magneschi, L., Subramanian, V., et al. (2011). Multiple facets of anoxic metabolism and hydrogen production in the unicellular green alga Chlamydomonas reinhardtii. New Phytol. 190, 279-288. doi: 10.1111/j.1469-8137.2010.03534.x

Guo, L., and Paiva, N. L. (1995). Molecular cloning and expression of alfalfa (Medicago sativa L.) vestitone reductase, the penultimate enzyme in medicarpin biosynthesis. Arch. Biochem. Biophys. 320, 353-360. doi: 10.1016/00039861(95)90019-5

Harris, E. H. (1989). The Chlamydomonas Sourcebook: A Comprehensive Guide to Biology and Laboratory Use. San Diego, CA: Academic Press.

Hua, C., Linling, L., Feng, X., Yan, W., Honghui, Y., Conghua, W., et al. (2013). Expression patterns of an isoflavone reductase-like gene and its possible roles in secondary metabolism in Ginkgo biloba. Plant Cell Rep. 32, 637-650. doi: $10.1007 /$ s00299-013-1397-2 
Jaeger, D., Hübner, W., Huser, T., Mussgnug, J. H., and Kruse, O. (2017). Nuclear transformation and functional gene expression in the oleaginous microalga Monoraphidium neglectum. J. Biotechnol. 249, 10-11. doi: 10.1016/j.jbiotec. 2017.03.011

Kavanagh, K. L., Jörnvall, H., Persson, B., and Oppermann, U. (2008). Mediumand short-chain dehydrogenase/reductase gene and protein families: the SDR superfamily: functional and structural diversity within a family of metabolic and regulatory enzymes. Cell Mol. Life Sci. 65, 3895-3906. doi: 10.1007/s00018-0088588-y

Kim, S. G., Kim, S. T., Wang, Y., Kim, S. K., Lee, C. H., Kim, K. K., et al. (2010). Overexpression of rice isoflavone reductase-like gene (OsIRL) confers tolerance to reactive oxygen species. Physiol. Plant. 138, 1-9. doi: 10.1111/j.1399-3054. 2009.01290.x

Kim, S. T., Cho, K. S., Kim, S. G., Kang, S. Y., and Kang, K. Y. (2003). A rice isoflavone reductase-like gene, OsIRL, is induced by rice blast fungal elicitor. Mol. Cells 16, 224-231.

Kindle, K. L. (1990). High-frequency nuclear transformation of Chlamydomonas reinhardtii. Proc. Natl. Acad. Sci. U.S.A. 87, 1228-1232. doi: 10.1073/pnas.87.3. 1228

Kopriva, S., and Rennenberg, H. (2004). Control of sulphate assimilation and glutathione synthesis: interaction with $\mathrm{N}$ and $\mathrm{C}$ metabolism. J. Exp. Bot. 55, 1831-1842. doi: 10.1093/jxb/erh203

Koprivova, A., Suter, M., den Camp, R. O., Brunold, C., and Kopriva, S. (2000). Regulation of sulfate assimilation by nitrogen in Arabidopsis. Plant Physiol. 122, 737-746. doi: 10.1104/pp.122.3.737

Kremers, G. J., Goedhart, J., van Munster, E. B., and Gadella, T. W. Jr. (2006). Cyan and yellow super fluorescent proteins with improved brightness, protein folding, and FRET Forster radius. Biochemistry 45, 6570-6580. doi: 10.1021/ bi0516273

Kruse, O., Rupprecht, J., Bader, K. P., Thomas-Hall, S., Schenk, P. M., Finazzi, G., et al. (2005). Improved photobiological $\mathrm{H}_{2}$ production in engineered green algal cells. J. Biol. Chem. 280, 34170-34177. doi: 10.1074/jbc.M503840200

Lauersen, K. J., Kruse, O., and Mussgnug, J. H. (2015). Targeted expression of nuclear transgenes in Chlamydomonas reinhardtii with a versatile, modular vector toolkit. Appl. Microbiol. Biotechnol. 99, 3491-3503. doi: 10.1007/s00253014-6354-7

Lers, A., Burd, S., Lomaniec, E., Droby, S., and Chalutz, E. (1998). The expression of a grapefruit gene encoding an isoflavone reductase-like protein is induced in response to UV irradiation. Plant Mol. Biol. 36, 847-856. doi: 10.1023/A: 1005996515602

Loreto, F., Barta, C., Brilli, F., and Nogues, I. (2006). On the induction of volatile organic compound emissions by plants as consequence of wounding or fluctuations of light and temperature. Plant Cell Environ. 29, 1820-1828. doi: 10.1111/j.1365-3040.2006.01561.x

Louie, G. V., Baiga, T. J., Bowman, M. E., Koeduka, T., Taylor, J. H., Spassova, S. M., et al. (2007). Structure and reaction mechanism of basil eugenol synthase. PLoS ONE 2:e993. doi: 10.1371/journal.pone.0000993

Lubitz, W., Ogata, H., Rudiger, O., and Reijerse, E. (2014). Hydrogenases. Chem Rev. 114, 4081-4148. doi: 10.1021/cr4005814

Mano, J., Belles-Boix, E., Babiychuk, E., Inze, D., Torii, Y., Hiraoka, E., et al. (2005). Protection against photooxidative injury of tobacco leaves by 2 -alkenal reductase. Detoxication of lipid peroxide-derived reactive carbonyls. Plant Physiol. 139, 1773-1783. doi: 10.1104/pp.105.070391

Maxwell, K., and Johnson, G. N. (2000). Chlorophyll fluorescence-a practical guide. J. Exp. Bot. 51, 659-668. doi: 10.1093/jxb/51.345.659

May, P., Wienkoop, S., Kempa, S., Usadel, B., Christian, N., Rupprecht, J., et al. (2008). Metabolomics- and proteomics-assisted genome annotation and analysis of the draft metabolic network of Chlamydomonas reinhardtii. Genetics 179, 157-166. doi: 10.1534/genetics.108.088336

Melis, A., Zhang, L., Forestier, M., Ghirardi, M. L., and Seibert, M. (2000). Sustained photobiological hydrogen gas production upon reversible inactivation of oxygen evolution in the green alga Chlamydomonas reinhardtii. Plant Physiol. 122, 127-136. doi: 10.1104/pp.122.1.127

Metz, J. G., Pakrasi, H. B., Seibert, M., and Arntzer, C. J. (1986). Evidence for a dual function of the herbicide-binding D1 protein in photosystem II. FEBS Lett. 205, 269-274. doi: 10.1016/0014-5793(86)80911-5

Miller, R., Wu, G., Deshpande, R. R., Vieler, A., Gartner, K., Li, X., et al. (2010). Changes in transcript abundance in Chlamydomonas reinhardtii following nitrogen deprivation predict diversion of metabolism. Plant Physiol. 154, 17371752. doi: 10.1104/pp.110.165159

Min, T., Kasahara, H., Bedgar, D. L., Youn, B., Lawrence, P. K., Gang, D. R., et al. (2003). Crystal structures of pinoresinol-lariciresinol and phenylcoumaran benzylic ether reductases and their relationship to isoflavone reductases. J. Biol. Chem. 278, 50714-50723. doi: 10.1074/jbc.M308493200

Molnar, A., Bassett, A., Thuenemann, E., Schwach, F., Karkare, S., Ossowski, S., et al. (2009). Highly specific gene silencing by artificial microRNAs in the unicellular alga Chlamydomonas reinhardtii. Plant J. 58, 165-174. doi: 10.1111/ j.1365-313X.2008.03767.x

Mosblech, A., Feussner, I., and Heilmann, I. (2009). Oxylipins: structurally diverse metabolites from fatty acid oxidation. Plant Physiol. Biochem. 47, 511-517. doi: 10.1016/j.plaphy.2008.12.011

Moummou, H., Kallberg, Y., Tonfack, L. B., Persson, B., and van der Rest, B. (2012). The plant short-chain dehydrogenase (SDR) superfamily: genome-wide inventory and diversification patterns. BMC Plant Biol. 12:219. doi: 10.1186/ 1471-2229-12-219

Mueller, M. J., and Berger, S. (2009). Reactive electrophilic oxylipins: pattern recognition and signalling. Phytochemistry 70, 1511-1521. doi: 10.1016/j. phytochem.2009.05.018

Muench, M., Hsin, C. H., Ferber, E., Berger, S., and Mueller, M. J. (2016). Reactive electrophilic oxylipins trigger a heat stress-like response through HSFA1 transcription factors. J. Exp. Bot. 67, 6139-6148. doi: 10.1093/jxb/ erw376

Nelson, J. A., Savereide, P. B., and Lefebvre, P. A. (1994). The CRY1 gene in Chlamydomonas reinhardtii: structure and use as a dominant selectable marker for nuclear transformation. Mol. Cell. Biol. 14, 4011-4019. doi: 10.1128/MCB. 14.6.4011

Neupert, J., Karcher, D., and Bock, R. (2009). Generation of Chlamydomonas strains that efficiently express nuclear transgenes. Plant J. 57, 1140-1150. doi: 10.1111/j.1365-313X.2008.03746.x

Nguyen, A. V., Toepel, J., Burgess, S., Uhmeyer, A., Blifernez, O., Doebbe, A., et al. (2011). Time-course global expression profiles of Chlamydomonas reinhardtii during photo-biological $\mathrm{H}_{2}$ production. PLOS ONE 6:e29364. doi: 10.1371/ journal.pone.0029364

Organisation for Economic Co-operation and Development [OECD]/International Energy Agency [IEA] (2011). OECD Green Growth Studies: Energy. Available at: http://www.oecd.org/greengrowth/greeningenergy/49157219.pdf

Paiva, N. L., Edwards, R., Sun, Y., Hrazdina, G., and Dixon, R. A. (1991). Stress responses in alfalfa (Medicago sativa L.) 11. Molecular cloning and expression of alfalfa isoflavone reductase, a key enzyme of isoflavonoid phytoalexin biosynthesis. Plant Mol. Biol. 17, 653-667. doi: 10.1007/BF00037051

Persson, B., Kallberg, Y., Bray, J. E., Bruford, E., Dellaporta, S. L., Favia, A. D., et al. (2009). The SDR (short-chain dehydrogenase/reductase and related enzymes) nomenclature initiative. Chem. Biol. Interact. 178, 94-98. doi: 10.1016/j.cbi. 2008.10.040

Persson, B., Kallberg, Y., Oppermann, U., and Jornvall, H. (2003). Coenzymebased functional assignments of short-chain dehydrogenases/reductases (SDRs). Chem. Biol. Interact. 14, 271-278. doi: 10.1016/S0009-2797(02) 00223-5

Petrucco, S., Bolchi, A., Foroni, C., Percudani, R., Rossi, G. L., and Ottonello, S. (1996). A maize gene encoding an NADPH binding enzyme highly homologous to isoflavone reductases is activated in response to sulfur starvation. Plant Cell 8, 69-80. doi: 10.1105/tpc.8.1.69

Pfaffl, M. W. (2001). A new mathematical model for relative quantification in real-time RT-PCR. Nucleic Acids Res. 29:e45. doi: 10.1093/nar/ 29.9.e45

Roach, T., Baur, T., Stoggl, W., and Krieger-Liszkay, A. (2017). Chlamydomonas reinhardtii responding to high light: a role for 2-propenal (acrolein). Physiol. Plant. doi: 10.1111/ppl.12567 [Epub ahead of print].

Salbitani, G., Vona, V., Bottone, C., Petriccione, M., and Carfagna, S. (2015). Sulfur deprivation results in oxidative perturbation in Chlorella sorokiniana (211/8k). Plant Cell Physiol. 56, 897-905. doi: 10.1093/pcp/pcv015

Schönfeld, C., Wobbe, L., Borgstadt, R., Kienast, A., Nixon, P. J., and Kruse, O. (2004). The nucleus-encoded protein MOC1 is essential for mitochondrial light acclimation in Chlamydomonas reinhardtii. J. Biol. Chem. 279, 50366-50374. doi: 10.1074/jbc.M408477200 
Scoma, A., Durante, L., Bertin, L., and Fava, F. (2014). Acclimation to hypoxia in Chlamydomonas reinhardtii: Can biophotolysis be the major trigger for long-term $\mathrm{H}_{2}$ production? New Phytol. 204, 890-900. doi: 10.1111/nph.12964

Shoji, T., Winz, R., Iwase, T., Nakajima, K., Yamada, Y., and Hashimoto, T. (2002). Expression patterns of two tobacco isoflavone reductase-like genes and their possible roles in secondary metabolism in tobacco. Plant Mol. Biol. 50, 427-440. doi: 10.1023/A:1019867732278

Steinbeck, J., Nikolova, D., Weingarten, R., Johnson, X., Richaud, P., Peltier, G., et al. (2015). Deletion of proton gradient regulation 5 (PGR5) and PGR5Like 1 (PGRL1) proteins promote sustainable light-driven hydrogen production in Chlamydomonas reinhardtii due to increased PSII activity under sulfur deprivation. Front. Plant Sci. 6:892. doi: 10.3389/fpls.2015.00892

Tardif, M., Atteia, A., Specht, M., Cogne, G., Rolland, N., Brugiere, S., et al. (2012). PredAlgo: a new subcellular localization prediction tool dedicated to green algae. Mol. Biol. Evol. 29, 3625-3639. doi: 10.1093/molbev/mss178

Toepel, J., Illmer-Kephalides, M., Jaenicke, S., Straube, J., May, P., Goesmann, A., et al. (2013). New insights into Chlamydomonas reinhardtii hydrogen production processes by combined microarray/RNA-seq transcriptomics. Plant Biotechnol. J. 11, 717-733. doi: 10.1111/pbi.12062

Uhmeyer, A., Ballottari, M., Cecchin, M., and Wobbe, L. (2017). Impaired mitochondrial transcription termination disrupts the stromal redox poise in Chlamydomonas. Plant Physiol. 174, 1399-1419. doi: 10.1104/pp.16.00946

Vass, I., Styring, S., Hundal, T., Koivuniemi, A., Aro, E., and Andersson, B. (1992). Reversible and irreversible intermediates during photoinhibition of photosystem II: stable reduced QA species promote chlorophyll triplet formation. Proc. Natl. Acad. Sci. U.S.A. 89, 1408-1412. doi: 10.1073/pnas.89. 4.1408

Volgusheva, A., Styring, S., and Mamedov, F. (2013). Increased photosystem II stability promotes $\mathrm{H} 2$ production in sulfur-deprived Chlamydomonas reinhardtii. Proc. Natl. Acad. Sci. U.S.A. 110, 7223-7228. doi: 10.1073/pnas. 1220645110

Volgusheva, A. A., Zagidullin, V. E., Antal, T. K., Korvatovsky, B. N., Krendeleva, T. E., Paschenko, V. Z., et al. (2007). Examination of chlorophyll fluorescence decay kinetics in sulfur deprived algae Chlamydomonas reinhardtii. Biochim. Biophys. Acta 1767, 559-564. doi: 10.1016/j.bbabio.2007.04.006

Wykoff, D. D., Davies, J. P., Melis, A., and Grossman, A. R. (1998). The regulation of photosynthetic electron transport during nutrient deprivation in Chlamydomonas reinhardtii. Plant Physiol. 117, 129-139. doi: 10.1104/pp.117. 1.129

Yamauchi, Y., Hasegawa, A., Taninaka, A., Mizutani, M., and Sugimoto, Y. (2011). NADPH-dependent reductases involved in the detoxification of reactive carbonyls in plants. J. Biol. Chem. 286, 6999-7009. doi: 10.1074/jbc.M110. 202226

Yamauchi, Y., Kunishima, M., Mizutani, M., and Sugimoto, Y. (2015). Reactive short-chain leaf volatiles act as powerful inducers of abiotic stress-related gene expression. Sci. Rep. 5:8030. doi: 10.1038/srep08030

Zhang, L., Happe, T., and Melis, A. (2002). Biochemical and morphological characterization of sulfur-deprived and $\mathrm{H}_{2}$-producing Chlamydomonas reinhardtii (green alga). Planta 214, 552-561. doi: 10.1007/s0042501 00660

Zheng, H.-Q., Chiang-Hsieh, Y.-F., Chien, C.-H., Hsu, B.-K. J., Liu, T.-L., Chen, C.N. N., et al. (2014). AlgaePath: comprehensive analysis of metabolic pathways using transcript abundance data from next-generation sequencing in green algae. BMC Genomics 15:196. doi: 10.1186/1471-2164-15-196

Conflict of Interest Statement: The authors declare that the research was conducted in the absence of any commercial or financial relationships that could be construed as a potential conflict of interest.

Copyright (c) 2017 Venkanna, Südfeld, Baier, Homburg, Patel, Wobbe and Kruse. This is an open-access article distributed under the terms of the Creative Commons Attribution License (CC BY). The use, distribution or reproduction in other forums is permitted, provided the original author(s) or licensor are credited and that the original publication in this journal is cited, in accordance with accepted academic practice. No use, distribution or reproduction is permitted which does not comply with these terms. 